DFTT $81 / 95$

NORDITA 95/85-P

January 1996

\title{
String techniques for the calculation of renormalization constants in field theory
}

\author{
Paolo Di Vecchia and Lorenzo Magnea!, \\ NORDITA \\ Blegdamsvej 17, DK-2100 Copenhagen Ø, Denmark \\ Alberto Lerdaf and Rodolfo Russo, \\ Dipartimento di Scienze e Tecnologie Avanzate and \\ Dipartimento di Fisica Teorica, Università di Torino \\ and I.N.F.N., Sezione di Torino \\ Via P.Giuria 1, I-10125 Torino, Italy \\ Raffaele Marotta \\ Dipartimento di Scienze Fisiche, Università di Napoli \\ and I.N.F.N., Sezione di Napoli \\ Mostra D'Oltremare, Pad. 19, I-80125 Napoli, Italy
}

\begin{abstract}
We describe a set of methods to calculate gauge theory renormalization constants from string theory, all based on a consistent prescription to continue off shell open bosonic string amplitudes. We prove the consistency of our prescription by explicitly evaluating the renormalizations of the two, three and four-gluon amplitudes, and showing that they obey the appropriate Ward identities. The field theory limit thus performed corresponds to the background field method in Feynman gauge. We identify precisely the regions in string moduli space that correspond to different classes of Feynman diagrams, and in particular we show how to isolate contributions to the effective action. Ultraviolet divergent terms are then encoded in a single string integral over the modular parameter $\tau$. Finally, we derive a multiloop expression for the effective action by computing the partition function of an open bosonic string interacting with an external non-abelian background gauge field.
\end{abstract}

\footnotetext{
*e-mail: DIVECCHIA@nbivax.nbi.dk

${ }^{\dagger}$ On leave from Università di Torino, Italy

${ }^{\ddagger}$ II Facoltà di Scienze M.F.N., Università di Torino (sede di Alessandria), Italy
} 


\section{Introduction}

String theories, unlike field theories, contain a physical dimensional parameter, the Regge slope $\alpha^{\prime}$, that acts as an ultraviolet cutoff in the integrals over loop momenta, making multiloop amplitudes free from ultraviolet divergences. This is a basic reason why a string theory can provide a consistent perturbative quantum theory of gravity, unified with non-abelian gauge theories.

Another useful feature of string theory is the fact that, at each order of string perturbation theory, one does not get the large proliferation of diagrams characteristic of field theories, which makes it extremely difficult to perform high order calculations. In the case of closed strings one gets only one diagram at each order, while in the open string the number of diagrams remains small. Furthermore, the expression of these diagrams is known explicitly, in the case of the bosonic string, for an arbitrary perturbative order, including also the measure of integration on moduli space [1].

Finally, it is well known that in the limit of infinite string tension $\left(\alpha^{\prime} \rightarrow 0\right)$ string theories reduce to non-abelian gauge theories, unified with gravity, order by order in perturbation theory. This means, in particular, that in this limit one must reproduce, order by order, $S$-matrix elements, ultraviolet divergences, and all other physical quantities that one computes in perturbative non-abelian gauge theories.

The combination of these different features of string theory has led several authors [2, 3, 4, 5, 6, 7] to show that in many cases string theory can be an efficient conceptual and computational tool in different areas of perturbative field theory. In particular, because of the compactness of the multiloop string expression, it is easier to calculate non-abelian gauge theory amplitudes by starting from a string theory, and performing the zero slope limit, rather than using traditional techniques. In this way the one-loop amplitude involving four external gluons has been computed, reproducing the known field theoretical result with much less computational cost [8]. Following the same approach, also the one-loop five-gluon amplitude has been computed for the first time [9].

As observed in Ref. [10], in order to extract field theory results from string theory, it is not necessary to use a consistent string theory. It can be sufficient to use the simplest string theory that contains the desired field theory in the zero slope limit (the open bosonic string if one is interested in non-abelian gauge theories). String theory in fact proves itself to be a rather versatile and robust tool: as we shall see, one recovers correct results in the field theory limit even when the consistency of the full string theory has been severely damaged, for example by continuing the results to arbitrary space-time dimension, and by extending off shell the string amplitudes, which implies a breaking of conformal invariance. Similar observations have led to the conjecture that the string organization may be understood entirely in terms of a field theory analysis, and in fact in some instances this has been shown [11, 12, 13]. However, in view of a possible multiloop generalization of the 
known results [14], we believe that further input from string theory may prove necessary.

In a previous letter 15 we have considered the open bosonic string with ChanPaton factors, and we have computed the one-loop two-gluon amplitude, with a suitable off-shell extrapolation in order to get rid of infrared divergences. By performing then the zero slope limit, we have obtained the gluon wave function renormalization constant $Z_{A}$, which turned out to coincide with the one calculated in the background field method.

In this paper we extend our procedure to the three and four-gluon amplitudes, and thus compute also the renormalization constants $Z_{3}$, for the three-gluon vertex, and $Z_{4}$, for the four-gluon vertex. This is a test of the consistency of our off-shell prescription, as we can show the validity of the Ward identities $Z_{A}=Z_{3}=Z_{4}$, that are characteristic of the background field method.

The information obtained with our method complements the knowledge of how gauge-invariant on-shell scattering amplitudes can be derived from string theory. In fact, in field theory, one is in general interested in calculating off-shell, gaugedependent quantities such as anomalous dimensions or general Green functions. An alternative set of rules for calculating these objects is useful only if the choice of gauge, regularization and renormalization prescriptions is uniquely specified. The difficulty with string theory is of course that all one starts with is a prescription to calculate scattering amplitudes, which is only valid on shell. Different off-shell continuations must be shown to be consistent, and may lead to different gauges or renormalization schemes. To date, the only known consistent prescription for the off-shell continuation of string amplitudes 20 leads to a vanishing wave-function renormalization for the gauge field, in apparent contradiction with the results of a detailed analysis of on-shell amplitudes [11], which implies that string theory leads to a combination of the background field method with the non-linear GervaisNeveu gauge. In the following we will solve this puzzle by proposing a different, and simpler, prescription for the off-shell continuation of the amplitudes, which leads unambiguously to the background field method.

The calculation of renormalization constants can be performed using three different, although closely related methods. The most straightforward method, in the spirit of [6], is based on the evaluation of the field theory limit of the full string amplitude, from which ultraviolet divergences can be consistently extracted after off-shell continuation. A careful analysis of the string amplitudes leads to a precise identification of the regions in moduli space that correspond to different classes of Feynman diagrams in field theory. Having learnt how to identify one-particle irreducible diagrams, contributing to the effective action, one may look for a method to isolate these contributions directly in the general expression for the amplitude. This is achieved by using a different expression for the bosonic world-sheet Green function, along the lines of [2]. We establish a precise relationship between these two methods, showing explicitly how the contributions to the effective action are picked out of the full string amplitude. Finally, having observed how string ampli- 
tudes seem to be intimately connected with the background field method, we go on to show how effective actions with arbitrary numbers of legs and loops can be compactly represented in terms of a generating functional describing the propagation of an open string in an external non-abelian gauge field. The renormalization constants derived from this generating functional coincide with those previously obtained calculating string scattering amplitudes.

The first method (presented in Sections 3, 4 and 5) uses the open bosonic string amplitudes for two, three and four gluons, suitably continued off shell. The amplitudes are expressed as an integral over the puncture coordinates, representing the locations of the insertions of the gluon operators on the open string boundaries, and over the modular parameter of the annulus, $\tau$. The integral over the punctures has a Laurent expansion in terms of the variable $k=e^{-2 \tau}$. The leading term of this expansion, of order $k^{-1}$, corresponds to tachyon exchange in the loop, and must be discarded by hand. The next-to-leading term, of order 1, corresponds to gluon exchange in the loop, and gives the field theory limit. Higher order contributions, behaving as $k^{n}$ with $n>0$, are negligible in the field theory limit. If we then concentrate on the term of order 1 , we see that the infinities appearing in the field theory limit arise from different regions of integration over the punctures. There are basically two kinds of contributions [8]. The first one is obtained by expanding the integrand for large $\tau$, isolating the term of order 1 in $k$, and then integrating the resulting expression over the punctures. This contribution corresponds to oneparticle irreducible diagrams in field theory. The second kind of contribution arises instead from regions of integration in which some punctures are taken to be very close to each other. This is often referred to as the pinching limit, and corresponds to field theory diagrams that are connected but not one-particle irreducible. The sum of the two contributions reproduces the infinities of the full connected Green functions, truncated with free propagators and suitably continued off shell.

The second method is based on the use of an alternative form of the bosonic Green function, proposed in Ref. [16], in which the logarithmic singularity associated with the pinching limit is regularized by means of a $\zeta$-function regularization. Using this regularized expression for the Green function simplifies considerably the evaluation of renormalization constants. In fact, as we shall see, having eliminated the reducible diagrams associated with the pinching regions, one can easily isolate ultraviolet divergent contributions by focusing on the terms in the integrand with the appropriate power of the modular parameter. It is then possible to neglect the exponentials of the bosonic Green functions that constitute the bulk of the integrand. This simplifies the calculation to the point that it is actually possible to perform the integrals over the punctures exactly, before the field theory limit is taken.

Finally, we show how all divergent contributions to the effective action can be generated from a single functional, describing the interaction of an open bosonic string with an external non-abelian background gauge field. Using this formalism, one sees the connection between string theory and the background field method 
from a different angle. String-derived gluon amplitudes without pinchings can be understood as scattering amplitudes in a background field constructed out of plane waves. Contributions to the effective action can be isolated by focusing on slowly varying background fields, and are then connected to gluon amplitudes for low energy gluons. Manipulating the expression for the all-order partition function of the string in such a slowly varying background, we can formally generalize the calculation of the effective action, and we obtain a compact expression for the string integral generating the renormalization constants, valid for any number of string loops.

The paper is organized as follows. In Section 2 we consider the open bosonic string, and we write the explicit expression of the $M$-gluon amplitude at $h$ loops, including the overall normalization. We specialize then to the case of tree and one-loop diagrams, detailing various choices of integration variables. In Section 3 we sketch the calculation of the one-loop two-gluon amplitude, already presented in [15], and we show how to extract the gluon wave function renormalization constant $Z_{A}$. In Section 4 we extend our previous calculation to the one-loop three-gluon amplitude, and we discuss in detail the various regions of integration over the punctures that contribute to the field theory limit. In particular, we show how one can identify at the string level the classes of Feynman diagrams that correspond to each such region, and we derive the renormalization constant $Z_{3}$. In Section 5 we consider the one-loop four-gluon amplitude, we extract the renormalization constant $Z_{4}$, and we discuss some subtleties arising in the treatment of contact terms. In Section 6 we show how, using a regularized expression of the Green function, one is naturally led to calculate contributions to the effective action, and finally in Section 7 we recover and extend these results by computing the partition function of a string interacting with an external non-abelian field. Section 8 summarizes our results and compares the different approaches, having in mind possible applications to multiloop calculations in gauge theories.

\section{The $M$-gluon amplitude}

The properly normalized (connected) $S$-matrix element describing the scattering of $M$ string states $\left|\alpha_{1}\right\rangle, \ldots,\left|\alpha_{M}\right\rangle$ with momenta $p_{1}, \ldots, p_{M}$ is

$$
S\left(\alpha_{1}, \ldots, \alpha_{M}\right)=\mathrm{i}(2 \pi)^{d} \delta^{(d)}\left(p_{1}+\ldots+p_{M}\right) A\left(\alpha_{1}, \ldots, \alpha_{M}\right) \prod_{i=1}^{M}\left(2 E_{i}\right)^{-1 / 2}
$$

where $d$ is the dimension of the target space-time with metric $(-,+, \ldots,+), E_{i}=$ $\left|p_{i}^{0}\right|$ is the energy of the $i$-th particle and $A\left(\alpha_{1}, \ldots, \alpha_{M}\right)$ is the $M$-point amplitude in which all propagators of the $M$ external states have been removed. Borrowing the terminology from field theory, we call $A\left(\alpha_{1}, \ldots, \alpha_{M}\right)$ the (unrenormalized) connected $M$-point amplitude. In string theory, these amplitudes can be computed 
pertubatively, and are written as

$$
\begin{aligned}
A\left(\alpha_{1}, \ldots, \alpha_{M}\right) & =\sum_{h=0}^{\infty} A^{(h)}\left(\alpha_{1}, \ldots, \alpha_{M}\right) \\
& =\sum_{h=0}^{\infty} g_{s}^{2 h-2} \hat{A}^{(h)}\left(\alpha_{1}, \ldots, \alpha_{M}\right)
\end{aligned}
$$

where $g_{s}$ is a dimensionless string coupling constant, which is introduced to formally control the perturbative expansion. In Eq. (2.2), $A^{(h)}$ represents the $h$-loop contribution. In particular, for the open bosonic string, $A^{(0)}$ corresponds to the scattering of string states inserted on the boundary of a disk, while $A^{(1)}$ corresponds to the scattering of states inserted either on one of the boundaries of an annulus (planar diagram), or on the boundary of a Moebius strip (non-orientable diagram), or on both boundaries of an annulus (non-planar diagram). Similarly the higher loop amplitudes $A^{(h)}$ with $h \geq 2$ correspond to the scattering of states inserted on the various boundaries of an open Riemann surface with a more complicated topology. In what follows we will limit our discussion to planar diagrams, but our analysis can be extended to the other cases as well.

An efficient way to explicitly obtain $A^{(h)}\left(\alpha_{1}, \ldots, \alpha_{M}\right)$ is to use the $M$-point $h$ loop vertex $V_{M ; h}$ of the operator formalism [1], which can be regarded as a generating functional for scattering amplitudes among arbitrary string states, at all orders in perturbation theory. In fact, by saturating the operator $V_{M ; h}$ with $M$ states $\left|\alpha_{1}\right\rangle, \ldots,\left|\alpha_{M}\right\rangle$, one obtains the corresponding amplitude,

$$
A^{(h)}\left(\alpha_{1}, \ldots, \alpha_{M}\right)=V_{M ; h}\left|\alpha_{1}\right\rangle \cdots\left|\alpha_{M}\right\rangle
$$

The explicit expression of $V_{M ; h}$ for the planar diagrams of the open bosonic string can be found in Ref. [1]. That expression still has to be properly normalized, and the correct normalization factor $C_{h}$ is given by

$$
C_{h}=\frac{1}{(2 \pi)^{d h}} g_{s}^{2 h-2} \frac{1}{\left(2 \alpha^{\prime}\right)^{d / 2}},
$$

where $\alpha^{\prime}$ is the inverse of the string tension (we refer to Appendix A for a derivation of this formula).

The vertex $V_{M ; h}$ depends on $M$ real Koba-Nielsen variables $z_{i}$ through $M$ projective transformations $V_{i}(z)$, which define local coordinate systems vanishing around each $z_{i}$, i.e. such that

$$
V_{i}^{-1}\left(z_{i}\right)=0
$$

When $V_{M ; h}$ is saturated with $M$ physical string states satisfying the mass-shell condition, the corresponding amplitude does not depend on the $V_{i}$ 's. However, as we discussed in Ref. [15], to extract information about the ultraviolet divergences that arise when the field theory limit is taken, it is necessary to relax the mass-shell condition, so that also the amplitudes $A^{(h)}$ will depend on the choice of projective 
transformations $V_{i}$ 's, just like the vertex $V_{M ; h}$. This is reminiscent of a well-known fact in gauge theories, namely that on-shell amplitudes are gauge invariant, whilst their off-shell counterparts are not. Indeed, one can regard the freedom of choosing the projective transformations $V_{i}$ 's as a sort of gauge freedom.

Let us now specialize to the case in which all $M$ states are gluons, and let us denote by $|p, \varepsilon\rangle$ a state representing a gluon with momentum $p$ and polarization $\varepsilon$. Such a state is created by the vertex operator

$$
V(z)=\mathrm{i} \mathcal{N}_{0}: \varepsilon \cdot \partial_{z} X(z) \mathrm{e}^{\mathrm{i} \sqrt{2 \alpha^{\prime}} p \cdot X(z)}:,
$$

where colons denote the standard normal ordering on the modes of the open string coordinate $X(z)$, and $\mathcal{N}_{0}$ is a normalization factor given by

$$
\mathcal{N}_{0}=g_{d} \sqrt{2 \alpha^{\prime}}
$$

with $g_{d}$ being the gauge coupling constant of the target space Yang-Mills theory. The latter is related to the dimensionless string coupling constant $g_{s}$ of Eq. (2.2) by

$$
g_{s}=\frac{g_{d}}{2}\left(2 \alpha^{\prime}\right)^{1-d / 4},
$$

as described in Appendix A. Notice that the vertex in Eq. (2.6) does not depend on the color index of the gluon, and thus the gauge group structure must be recovered via the Chan-Paton procedure.

If we write as usual

$$
X^{\mu}(z)=\hat{q}^{\mu}-\mathrm{i} \hat{p}^{\mu} \log z+\mathrm{i} \sum_{n \neq 0} \frac{\hat{a}_{n}^{\mu}}{n} z^{-n},
$$

then the gluon state is

$$
|p, \varepsilon\rangle \equiv \lim _{z \rightarrow 0} V(z)|0\rangle=\mathcal{N}_{0} \varepsilon \cdot \hat{a}_{-1}|p\rangle
$$

where $|p\rangle$ is the ground state with momentum $p$. The gluon is physical if

$$
\begin{aligned}
p^{2} & =0 \\
\varepsilon \cdot p & =0
\end{aligned}
$$

We will always enforce the transversality condition (2.12), while in discussing the field theory limit of the string amplitudes we will relax the mass-shell condition (2.11) (see also [15]). Relaxing also the transversality condition would just lead to more cumbersome expressions without changing the final results.

If we saturate the vertex $V_{M ; h}$ with $M$ gluon states and multiply the result with the appropriate Chan-Paton factor, we obtain the color-ordered $M$-gluon planar amplitude at $h$ loops, which we denote by $A_{P}^{(h)}\left(p_{1}, \ldots, p_{M}\right)$. If we pick the gauge 
group $S U(N)$, use transverse gluons, but choose not to enforce the mass-shell condition, we find

$$
\begin{aligned}
A_{P}^{(h)}\left(p_{1}, \ldots, p_{M}\right)= & N^{h} \operatorname{Tr}\left(\lambda^{a_{1}} \ldots \lambda^{a_{M}}\right) C_{h} \mathcal{N}_{0}^{M} \\
& \times \int[d m]_{h}^{M}\left\{\prod_{i<j}\left[\frac{\exp \left(\mathcal{G}^{(h)}\left(z_{i}, z_{j}\right)\right)}{\sqrt{V_{i}^{\prime}(0) V_{j}^{\prime}(0)}}\right]^{2 \alpha^{\prime} p_{i} \cdot p_{j}}\right. \\
& \times \exp \left[\sum_{i \neq j} \sqrt{2 \alpha^{\prime}} p_{j} \cdot \varepsilon_{i} \partial_{z_{i}} \mathcal{G}^{(h)}\left(z_{i}, z_{j}\right)\right. \\
& \left.\left.+\frac{1}{2} \sum_{i \neq j} \varepsilon_{i} \cdot \varepsilon_{j} \partial_{z_{i}} \partial_{z_{j}} \mathcal{G}^{(h)}\left(z_{i}, z_{j}\right)\right]\right\}_{\text {m.l. }},
\end{aligned}
$$

where the subscript "m.l." stands for multilinear, meaning that only terms linear in each polarization should be kept. In Eq. (2.13), which can be considered the master formula of our approach, $N^{h} \operatorname{Tr}\left(\lambda^{a_{1}} \cdots \lambda^{a_{M}}\right)$ is the Chan-Paton factor appropriate for an $M$-gluon $h$-loop planar diagram, with the $\lambda$ 's being the generators of $S U(N)$ in the fundamental representation, normalized as

$$
\operatorname{Tr}\left(\lambda^{a} \lambda^{b}\right)=\frac{1}{2} \delta^{a b}
$$

Further, $\mathcal{G}^{(h)}\left(z_{i}, z_{j}\right)$ is the $h$-loop world-sheet bosonic Green function, and $[d m]_{h}^{M}$ is the measure of integration on moduli space for an open Riemann surface of genus $h$ with $M$ operator insertions on the boundary [1]. The Green function $\mathcal{G}^{(h)}\left(z_{i}, z_{j}\right)$ can be expressed as

$$
\mathcal{G}^{(h)}\left(z_{i}, z_{j}\right)=\log E^{(h)}\left(z_{i}, z_{j}\right)-\frac{1}{2} \int_{z_{i}}^{z_{j}} \omega^{\mu}\left(2 \pi \operatorname{Im} \tau_{\mu \nu}\right)^{-1} \int_{z_{i}}^{z_{j}} \omega^{\nu}
$$

where $E^{(h)}\left(z_{i}, z_{j}\right)$ is the prime-form, $\omega^{\mu}(\mu=1, \ldots, h)$ the abelian differentials and $\tau_{\mu \nu}$ the period matrix of an open Riemann surface of genus $h$. All these objects, as well as the measure on moduli space $[d m]_{h}^{M}$, can be explicitly written down in the Schottky parametrization of the Riemann surface, and their expressions for arbitrary $h$ can be found for example in [17]. Here we will only reproduce the explicit expression for the measure, to give a flavor of the ingredients that enter the full string theoretic calculations. It is

$$
\begin{aligned}
{[d m]_{h}^{M} } & =\frac{\prod_{i=1}^{M} d z_{i}}{d V_{a b c}} \prod_{\mu=1}^{h}\left[\frac{d k_{\mu} d \xi_{\mu} d \eta_{\mu}}{k_{\mu}^{2}\left(\xi_{\mu}-\eta_{\mu}\right)^{2}}\left(1-k_{\mu}\right)^{2}\right] \\
& \times\left[\operatorname{det}\left(-i \tau_{\mu \nu}\right)\right]^{-d / 2} \prod_{\alpha}^{\prime}\left[\prod_{n=1}^{\infty}\left(1-k_{\alpha}^{n}\right)^{-d} \prod_{n=2}^{\infty}\left(1-k_{\alpha}^{n}\right)^{2}\right] .
\end{aligned}
$$

Here $k_{\mu}$ are the multipliers and $\xi_{\mu}$ and $\eta_{\mu}$ the fixed points of the generators of the Schottky group; $d V_{a b c}$ is the projective invariant volume element

$$
d V_{a b c}=\frac{d \rho_{a} d \rho_{b} d \rho_{c}}{\left(\rho_{a}-\rho_{b}\right)\left(\rho_{a}-\rho_{c}\right)\left(\rho_{b}-\rho_{c}\right)},
$$


where $\rho_{a}, \rho_{b}, \rho_{c}$ are any three of the $M$ Koba-Nielsen variables, or of the $2 h$ fixed points of the generators of the Schottky group, which can be fixed at will; finally, the primed product over $\alpha$ denotes a product over classes of elements of the Schottky group [17]. Notice that in the open string the Koba-Nielsen variables must be cyclically ordered, for example according to

$$
z_{1} \geq z_{2} \geq \cdots \geq z_{M}
$$

and the ordering of Koba-Nielsen variables automatically prescribes the ordering of color indices.

Clearly expressions such as Eq. (2.16) can be very difficult to handle in practice, as they involve infinite products (or sums) over subgroups of a discrete group. However, as we shall see, all such expressions simplify drastically in the field theory limit $\alpha^{\prime} \rightarrow 0$. In this limit, only a finite number of terms contribute to the products, and calculations remain manageable.

The reader may also have noticed that we have left the value of the spacetime dimension $d$ arbitrary, and we have not fixed it to the critical dimension, $d=26$. As we shall see, this flagrant violation of string consistency does not affect the field theory limit. This may be understood if we interpret $d$ as the number of uncompactified dimensions, and we assume that $d^{\prime}=26-d$ dimensions have been compactified. Of course the $d^{\prime}$ compactified dimensions will contribute to the string partition function and to the scattering amplitudes, but those contributions factorize, and, in the field theory limit, behave just as $d^{\prime}$ scalars coupled to the gauge field. The naïve procedure, simply letting $d$ be arbitrary, just corresponds to neglecting ab initio the contribution of these scalars.

For $h=0$ the situation is particularly simple. The Green function in Eq. 2.15) reduces to

$$
\mathcal{G}^{(0)}\left(z_{i}, z_{j}\right)=\log \left(z_{i}-z_{j}\right)
$$

while the measure $[d m]_{0}^{M}$ is simply

$$
[d m]_{0}^{M}=\frac{\prod_{i=1}^{M} d z_{i}}{d V_{a b c}} .
$$

Inserting Eq. (2.19) and Eq. (2.20) into Eq. (2.13), and writing explicitly all the normalization coefficients, we obtain the color-ordered, on-shell $M$-gluon amplitude at tree level ${ }^{1}$

$$
\begin{aligned}
A^{(0)}\left(p_{1}, \ldots, p_{M}\right) & =4 \operatorname{Tr}\left(\lambda^{a_{1}} \cdots \lambda^{a_{M}}\right) g_{d}^{M-2}\left(2 \alpha^{\prime}\right)^{M / 2-2} \\
& \times \int_{\Gamma_{0}} \frac{\prod_{i=1}^{M} d z_{i}}{d V_{a b c}}\left\{\prod_{i<j}\left(z_{i}-z_{j}\right)^{2 \alpha^{\prime} p_{i} \cdot p_{j}}\right.
\end{aligned}
$$

\footnotetext{
${ }^{1}$ We omit the subscript $P$ for planar, since at tree level only planar diagrams are present.
} 


$$
\left.\times \exp \left[\sum_{i<j}\left(\sqrt{2 \alpha^{\prime}} \frac{p_{j} \cdot \varepsilon_{i}-p_{i} \cdot \varepsilon_{j}}{\left(z_{i}-z_{j}\right)}+\frac{\varepsilon_{i} \cdot \varepsilon_{j}}{\left(z_{i}-z_{j}\right)^{2}}\right)\right]\right\}_{\text {m.l. }},
$$

where $\Gamma_{0}$ is the region identified by Eq. (2.18). Notice that any dependence on the local coordinates $V_{i}(z)$ drops out in the amplitude after enforcing the mass-shell condition. Eq. (2.21) is valid for $M \geq 3$, since the tree-level measure Eq. (2.20) is ill-defined for $M \leq 2$.

For $h=1$ the situation is more complicated but still manageable. In the Schottky representation, the annulus is described by a Schottky group generated by one real projective transformation $S$. This can be characterized by a multiplier $k$ and the attractive and repulsive fixed points $\xi$ and $\eta$. In terms of these variables, the measure $[d m]_{1}^{M}$ on moduli space turns out to be

$$
[d m]_{1}^{M}=\frac{\prod_{i=1}^{M} d z_{i}}{d V_{a b c}} d k d \xi d \eta \frac{1}{k^{2}(\xi-\eta)^{2}}\left(-\frac{1}{2 \pi} \ln k\right)^{-d / 2} \prod_{n=1}^{\infty}\left(1-k^{n}\right)^{2-d}
$$

where $d V_{a b c}$ is given again by Eq. (2.17), with $\rho_{a}, \rho_{b}$ and $\rho_{c}$ being any of the $M+2$ variables $\left\{z_{1}, \ldots, z_{M}, \xi, \eta\right\}$, which can be fixed at will. The remaining $M-1$ variables and $k$ are the moduli of the annulus with $M$ operator insertions on one boundary. In deriving Eq. (2.22) from Eq. (2.16), one should remember that the determinant of the period matrix, coming from the integration over the momenta circulating in the loops, can be expressed at one loop in terms of the multiplier of the Schottky generator as

$$
k=e^{2 \pi i \tilde{\tau}}
$$

Here, in the one-loop case, we have called the period matrix $\tilde{\tau}$ instead of $\tau$, as usually done, because later on we will use $\tau$ for a rescaled version of it (see Eq. (2.29)).

A crucial point that must be discussed before we proceed is the region $\Gamma_{1}$ over which the moduli must be integrated. One way of determining $\Gamma_{1}$ is to observe that the vertex $V_{M ; 1}$ can be obtained with the sewing procedure from $V_{M+2 ; 0}$ [18]. As we mentioned before, $V_{M+2 ; 0}$ depends on $M+2$ Koba-Nielsen variables ordered on the real line, as in Eq. (2.18). In the sewing process one identifies, say, legs $M+1$ and $M+2$ by means of a propagator $P(x)$ depending on a sewing parameter $x$, with $0<x<1$. The specific form of $P(x)$ is determined by the local coordinates $V_{i}(z)$ of the two legs that are sewn, in such a way that the factorizability and the cyclic properties of the vertex are maintained after sewing. We refer the reader to [18] for a thorough discussion of this point and of the sewing procedure in general. Here we simply want to point out that the two sets $\left\{z_{1}, \ldots, z_{M}, z_{M+1}, z_{M+2}\right\}$ and $\left\{z_{1}, \ldots, z_{M}, \xi, \eta\right\}$ are equivalent, and that we can establish a precise relation between the original Koba-Nielsen variables of the tree-level vertex $V_{M+2 ; 0}$, the fixed points $\xi$ and $\eta$, and the multiplier $k$ of the Schottky generator at one loop. To do so, we must use the explicit expression of the propagator $P(x)$ and of the chosen local coordinates $V_{i}(z)$. If we choose the Lovelace coordinates for $i=M+1, M+2$, and 
consequently the propagator $P(x)$ of Ref. [18], then a simple calculation leads to

$$
\begin{aligned}
k & =\frac{1-x}{x} \frac{\left(z_{1}-z_{M+1}\right)\left(z_{M}-z_{M+2}\right)}{\left(z_{1}-z_{M+2}\right)\left(z_{M}-z_{M+1}\right)}, \\
\xi & =\frac{z_{1}\left(z_{M+1}-z_{M+2}\right)-z_{M+1}\left(z_{1}-z_{M+2}\right) k}{\left(z_{M+1}-z_{M+2}\right)-\left(z_{1}-z_{M+2}\right) k}, \\
\eta & =z_{M+2} .
\end{aligned}
$$

Now we can exploit projective invariance to fix

$$
z_{1} \rightarrow 1 \quad, \quad z_{M+1} \rightarrow k \quad, \quad z_{M+2} \rightarrow 0 \quad,
$$

which clearly corresponds to

$$
\eta \rightarrow 0 \quad, \quad \xi \rightarrow \infty \quad
$$

With this choice, the measure in Eq. (2.22) becomes simply

$$
[d m]_{1}^{M}=\prod_{i=2}^{M} d z_{i} d k \frac{1}{k^{2}}\left(-\frac{1}{2 \pi} \ln k\right)^{-d / 2} \prod_{n=1}^{\infty}\left(1-k^{n}\right)^{2-d}
$$

while the integration region becomes

$$
1 \geq z_{2} \geq \cdots \geq z_{M} \geq k \geq 0
$$

This is the fundamental region $\Gamma_{1}$ over which the moduli $z_{2}, \ldots, z_{M}$ and $k$ must be integrated, with the measure given in Eq. (2.27).

We observed that the multiplier $k$ of the Schottky generator is related to the more commonly used modular parameter $\tilde{\tau}$ by Eq. (2.23). Since $k$ is real, $\tilde{\tau}$ is purely imaginary. Below, we will find it more convenient to replace it with the real variable

$$
\tau=-\mathrm{i} \pi \tilde{\tau}
$$

which varies between 0 and $\infty$. Similarly, instead of the Koba-Nielsen variables $z_{i}$, we will use the real variables

$$
\nu_{i}=-\frac{1}{2} \log z_{i}
$$

The integration region given by Eq. (2.28) becomes, in these variables

$$
0 \leq \nu_{2} \leq \cdots \leq \nu_{M} \leq \tau<\infty \quad .
$$

The one-loop bosonic Green function $\mathcal{G}^{(1)}\left(z_{i}, z_{j}\right)$ also simplifies considerably using Eqs. (2.25) and (2.26). It is given by

$$
\begin{aligned}
\mathcal{G}^{(1)}\left(z_{i}, z_{j}\right) & =\log \left(z_{i}-z_{j}\right)+\frac{1}{2 \log k}\left(\log \frac{z_{i}}{z_{j}}\right)^{2}+\log \left[\prod_{n=1}^{\infty} \frac{\left(1-k^{n} \frac{z_{j}}{z_{i}}\right)\left(1-k^{n} \frac{z_{i}}{z_{j}}\right)}{\left(1-k^{n}\right)^{2}}\right] \\
& =\log \left[-2 \pi \mathrm{i} \frac{\left.\theta_{1}\left(\frac{\mathrm{i}}{\pi}\left(\nu_{j}-\nu_{i}\right) \mid \frac{\mathrm{i}}{\pi} \tau\right)\right)}{\theta_{1}^{\prime}\left(0 \mid \frac{\mathrm{i}}{\pi} \tau\right)}\right]-\frac{\left(\nu_{j}-\nu_{i}\right)^{2}}{\tau}-\nu_{i}-\nu_{j} \quad, \quad
\end{aligned}
$$


where $\theta_{1}$ is the first Jacobi $\theta$ function. It is not difficult to show that

$$
z_{i} \partial_{z_{i}} \mathcal{G}^{(1)}\left(z_{i}, z_{j}\right)+z_{j} \partial_{z_{j}} \mathcal{G}^{(1)}\left(z_{i}, z_{j}\right)=1
$$

The function $\mathcal{G}^{(1)}\left(z_{i}, z_{j}\right)$ is related to the more commonly used one-loop Green function $G\left(\nu_{j}-\nu_{i}\right)$ of, say, Ref. [19, by

$$
G\left(\nu_{j}-\nu_{i}\right)=\mathcal{G}^{(1)}\left(z_{i}\left(\nu_{i}\right), z_{j}\left(\nu_{j}\right)\right)+\nu_{i}+\nu_{j} .
$$

Notice that $G\left(\nu_{j}-\nu_{i}\right)$ is a function only of the difference $\nu_{j}-\nu_{i}$, whereas $\mathcal{G}^{(1)}\left(z_{i}, z_{j}\right)$ does not have translational invariance in the $\nu$ plane, as is clear from Eq. (2.33). Furthermore, $G\left(\nu_{j}-\nu_{i}\right)$ is periodic on the annulus, i.e. $G(\nu)=G(\tau+\nu)$, reminding us that the points $\nu=0$ and $\nu=\tau$ represent the same physical location on the world sheet.

We are now in a position to write explicitly the $S U(N)$ color-ordered planar amplitude for $M \geq 2$ transverse gluons at one loop. It is

$$
\begin{aligned}
A_{P}^{(1)}\left(p_{1}, \ldots, p_{M}\right) & =N \operatorname{Tr}\left(\lambda^{a_{1}} \cdots \lambda^{a_{M}}\right) \frac{g_{d}^{M}}{(4 \pi)^{d / 2}}\left(2 \alpha^{\prime}\right)^{(M-d) / 2}(-1)^{M} \\
& \times \int_{\Gamma_{1}} \prod_{i=2}^{M} d \nu_{i} d \tau \mathrm{e}^{2 \tau} \tau^{-d / 2} \prod_{n=1}^{\infty}\left(1-\mathrm{e}^{-2 n \tau}\right)^{2-d} \\
& \times\left\{\prod_{i<j}\left[\sqrt{\frac{z_{i} z_{j}}{V_{i}^{\prime}(0) V_{j}^{\prime}(0)}} \exp \left(G\left(\nu_{j i}\right)\right)\right]^{2 \alpha^{\prime} p_{i} \cdot p_{j}}\right. \\
& \left.\times \exp \left[\sum_{i \neq j}\left(\sqrt{2 \alpha^{\prime}} p_{j} \cdot \varepsilon_{i} \partial_{i} G\left(\nu_{j i}\right)+\frac{1}{2} \varepsilon_{i} \cdot \varepsilon_{j} \partial_{i} \partial_{j} G\left(\nu_{j i}\right)\right)\right]\right\}_{\mathrm{m} .1 .},
\end{aligned}
$$

where $\nu_{j i} \equiv \nu_{j}-\nu_{i}$ and $\partial_{i} \equiv \partial / \partial \nu_{i}$. Notice that in the last square bracket of Eq. (2.35) we have been able to replace everywhere $\mathcal{G}$ with $G$, since the difference is proportional to terms that vanish because of the transversality condition (2.12). If we further enforce the mass-shell condition (2.11), any dependence on the local coordinates $V_{i}$ 's drops out. However, if we want to consider a formal off-shell extension of this amplitude, the dependence on the $V_{i}$ 's becomes relevant. Our attitude is to continue off shell the gluon momenta in an appropriate way, that we will discuss in the following sections, essentially setting

$$
p_{i}^{2}=m^{2},
$$

with $m^{2}$ acting as an infrared regulator. Then we regard the freedom of choosing the $V_{i}$ 's as a gauge freedom [15]. Clearly, the simplest situation occurs when the square root factors in Eq. (2.35) simplify, i.e. when

$$
V_{i}^{\prime}(0)=z_{i}
$$

With such a choice the amplitude in Eq. (2.35) coincides exactly with the one normally used [19], which is entirely written in terms of the translational invariant 
Green function $G(\nu)$. The conditions (2.5) and (2.37) are easily satisfied by choosing for example

$$
V_{i}(z)=z_{i} z+z_{i}
$$

This is our choice for this paper.

We conclude this section by deriving an alternative expression for the Green function in Eq. (2.34), that we will need in Section 6 .

Using Eq. (8.1.53) of Ref. [19], one can rewrite Eq. (2.34) as follows

$$
G\left(\nu_{j}-\nu_{i}\right)=\log \left[-\frac{2 \pi}{\log q} \sin \pi\left(\frac{\nu_{j}-\nu_{i}}{\tau}\right) \prod_{n=1}^{\infty} \frac{1-2 q^{2 n} \cos 2 \pi\left(\frac{\nu_{j}-\nu_{i}}{\tau}\right)+q^{4 n}}{\left(1-q^{2 n}\right)^{2}}\right],
$$

where $q=e^{-\pi^{2} / \tau}$. By means of the identity

$$
\log \left[1+b^{2}-2 b \cos x\right]=-2 \sum_{n=1}^{\infty} \frac{b^{n}}{n} \cos n x \quad,
$$

it is easy to put Eq. (2.39) in the form [16]

$$
G\left(\nu_{j}-\nu_{i}\right)=-\sum_{n=1}^{\infty} \frac{1+q^{2 n}}{n\left(1-q^{2 n}\right)} \cos 2 \pi n\left(\frac{\nu_{j}-\nu_{i}}{\tau}\right)+\ldots,
$$

where the dots stand for terms independent of $\nu_{i}$ and $\nu_{j}$, that will not be important in our discussion.

It is important to notice that the Green function in Eq. (2.34), or in Eq. (2.39), is logarithmically divergent in the limit $\nu_{i} \rightarrow \nu_{j}$. This singularity is essential to get, for example, the correct pole contributions in the various channels at tree level, as well as to extract, in the field theory limit, the contribution of one-particle reducible diagrams at one loop, as we will see in Sections 1 and 5. If we rewrite the Green function as in Eq. (2.41), this logarithmic singularity has not disappeared, but it manifests itself as a divergence in the sum.

In what follows we will show how, in the field theory limit, the one-loop gluon amplitudes derived from Eq. (2.35) develop ultraviolet divergences, and these divergences with our prescription reproduce exactly the results of the background field method in the Feynman gauge.

\section{The two-gluon amplitude}

Let us briefly recall the results for the two-gluon amplitude that we already presented in Ref. [15]. From Eq. (2.13) with $M=2$ we see that the color-ordered two-gluon amplitude at $h$ loops $(h \geq 1)$ is ${ }^{2}$

$$
A^{(h)}\left(p_{1}, p_{2}\right)=N^{h} \operatorname{Tr}\left(\lambda^{a_{1}} \lambda^{a_{2}}\right) C_{h} \mathcal{N}_{0}^{2} \int[d m]_{h}^{2}
$$

\footnotetext{
${ }^{2}$ Notice that this expression differs from the one in Eq. (5) of Ref. 15 by a term proportional to $\varepsilon_{1} \cdot p_{2} \varepsilon_{2} \cdot p_{1}$, which vanishes upon using momentum conservation and transversality.
} 


$$
\times\left[\frac{\exp \left(\mathcal{G}^{(h)}\left(z_{1}, z_{2}\right)\right)}{\sqrt{V_{1}^{\prime}(0) V_{2}^{\prime}(0)}}\right]^{2 \alpha^{\prime} p_{1} \cdot p_{2}} \varepsilon_{1} \cdot \varepsilon_{2} \partial_{z_{1}} \partial_{z_{2}} \mathcal{G}^{(h)}\left(z_{1}, z_{2}\right)
$$

where we dropped the subscript $P$, since for $S U(N)$ the planar diagram is the only one contributing to the two-point function. This formula generates, in the field theory limit, all the loop corrections to the Yang-Mills two-point function. In particular, as we showed in Ref. [15], $A^{(h)}\left(p_{1}, p_{2}\right)$ can be considered as a master formula containing all information necessary to compute the multiloop Yang-Mills beta function.

Let us now specialize to one loop $(h=1)$, using the explicit expressions written in the previous section, and in particular using $\nu_{i}$ as integration variables. We have

$$
\begin{aligned}
A^{(1)}\left(p_{1}, p_{2}\right) & =N \operatorname{Tr}\left(\lambda^{a_{1}} \lambda^{a_{2}}\right) \frac{g_{d}^{2}}{(4 \pi)^{d / 2}}\left(2 \alpha^{\prime}\right)^{2-d / 2} \\
& \times \int_{0}^{\infty} \mathcal{D} \tau \int_{0}^{\tau} d \nu f_{2}\left(\nu, \tau ; p_{1} \cdot p_{2}\right)
\end{aligned}
$$

where we introduced the notations

$$
\mathcal{D} \tau \equiv d \tau \mathrm{e}^{2 \tau} \tau^{-d / 2} \prod_{n=1}^{\infty}\left(1-\mathrm{e}^{-2 n \tau}\right)^{2-d}
$$

and

$$
f_{2}\left(\nu, \tau ; p_{1} \cdot p_{2}\right) \equiv-\frac{1}{2 \alpha^{\prime}} \varepsilon_{1} \cdot \varepsilon_{2} \mathrm{e}^{2 \alpha^{\prime} p_{1} \cdot p_{2} G(\nu)} \partial_{\nu}^{2} G(\nu)
$$

For later convenience we also define the integral (see Ref. [15])

$$
R(s) \equiv \frac{1}{\varepsilon_{1} \cdot \varepsilon_{2}} \frac{1}{s} \int_{0}^{\infty} \mathcal{D} \tau \int_{0}^{\tau} d \nu f_{2}(\nu, \tau ; s)
$$

Notice that if the mass shell condition (2.11) is enforced, the two-gluon amplitude becomes ill defined, as the kinematical prefactor vanishes, while the integral diverges. It is then necessary to somehow continue off shell the string amplitude, and then test that the results remain consistent in the field theory limit. This was first attempted in Ref. [20], where a prescription was found that leads to a vanishing wave function renormalization in the field theory limit. On the other hand, in Ref. [15] we have shown that, if we formally extend the gluon momenta off shell according to Eqs. (2.35) and (2.36), then in the limit $\alpha^{\prime} \rightarrow 0 A^{(1)}\left(p_{1}, p_{2}\right)$ develops an ultraviolet divergence, which in turn determines a non-vanishing wave function renormalization constant $Z_{A}$, equal to the one computed in the background field method.

In order to see this, we follow Ref. [8], and notice that the modular parameter $\tau$ and the coordinate $\nu$ are related to proper-time Schwinger parameters for the Feynman diagrams contributing to the two-point function. In particular, $t \sim \alpha^{\prime} \tau$ and $t_{1} \sim \alpha^{\prime} \nu$, where $t_{1}$ is the proper time associated with one of the two internal 
propagators, while $t$ is the total proper time around the loop. In the field theory limit, with the possible exception of pinching configurations, to be discussed later, these proper times have to remain finite, and thus the limit $\alpha^{\prime} \rightarrow 0$ must correspond to the limit $\{\tau, \nu\} \rightarrow \infty$ in the integrand. This can also be checked directly by examining the integral in Eq. (3.5), and is most easily seen by rescaling the integration variable according to $\hat{\nu} \equiv \nu / \tau$. The field theory limit is then determined by the asymptotic behavior of the Green function for large $\tau$, namely

$$
G(\nu)=-\frac{\nu^{2}}{\tau}+\log (2 \sinh (\nu))-4 \mathrm{e}^{-2 \tau} \sinh ^{2}(\nu)+O\left(e^{-4 \tau}\right)
$$

where $\nu$ must be taken to be finite; in this region, we may use

$$
G(\nu) \sim\left(\hat{\nu}-\hat{\nu}^{2}\right) \tau-\sum_{n=1}^{\infty} \frac{1}{n} \mathrm{e}^{-2 n \hat{\nu} \tau}-\mathrm{e}^{-2 \tau(1-\hat{\nu})}+2 \mathrm{e}^{-2 \tau}-\mathrm{e}^{-2 \tau(1+\hat{\nu})}
$$

so that

$$
\frac{\partial G}{\partial \nu} \sim 1-2 \hat{\nu}+2 \sum_{n=1}^{\infty} \mathrm{e}^{-2 n \hat{\nu} \tau}-2 \mathrm{e}^{-2 \tau(1-\hat{\nu})}+2 \mathrm{e}^{-2 \tau(1+\hat{\nu})}
$$

and

$$
\frac{\partial^{2} G}{\partial \nu^{2}} \sim-\frac{2}{\tau}-4 \sum_{n=1}^{\infty} n \mathrm{e}^{-2 n \hat{\nu} \tau}-4 \mathrm{e}^{-2 \tau(1-\hat{\nu})}-4 \mathrm{e}^{-2 \tau(1+\hat{\nu})} .
$$

In most cases it will be sufficient to keep only the first term in the infinite series appearing in Eqs. (3.7)-(3.9). The only exceptions are discussed in Section 5.

We now substitute these results into Eq. (3.2), and keep only terms that remain finite when $k=e^{-2 \tau} \rightarrow 0$; divergent terms are discarded by hand, since they correspond to the propagation of the tachyon in the loop. Notice that the divergence associated with the tachyon is not regularized by continuing the space-time dimension away from four, whereas all divergences associated with gluons are turned into the usual poles of dimensional regularization. Notice also that by taking the large $\nu$ limit with $\hat{\nu} \sim O(1)$ we have discarded two singular regions of integration that potentially contribute in the field theory limit, namely $\nu \rightarrow 0$ and $\nu \rightarrow \tau$. In these regions the Green function has a logarithmic singularity corresponding to the insertion of the two external states very close to each other, and this singularity, as we shall see the in the next section, in general gives non-vanishing contributions in the field theory limit. However, in the case of the two-gluon amplitude, these regions correspond to Feynman diagrams with a loop consisting of a single propagator, $i$. $e$. a "tadpole". Massless tadpoles are defined to vanish in dimensional regularization, and thus we are justified in discarding these contributions as well.

If we delete all unwanted contributions, replace the variable $\nu$ with $\hat{\nu} \equiv \nu / \tau$, and integrate by parts to remove the double derivative of the Green function, Eq. (3.5) becomes

$$
R(s)=\int_{0}^{\infty} d \tau \int_{0}^{1} d \hat{\nu} \tau^{1-d / 2} \mathrm{e}^{2 \alpha^{\prime} s\left(\hat{\nu}-\hat{\nu}^{2}\right) \tau}\left[(1-2 \hat{\nu})^{2}(d-2)-8\right]
$$


with $s=p_{1} \cdot p_{2}$. Integration by parts is not really necessary, but it makes calculations easier. The integral is now elementary, and yields

$$
R(s)=-\Gamma\left(2-\frac{d}{2}\right)\left(-2 \alpha^{\prime} s\right)^{d / 2-2} \frac{6-7 d}{1-d} B\left(\frac{d}{2}-1, \frac{d}{2}-1\right)
$$

where $B$ is the Euler beta function.

If we now substitute Eq. (3.11) into Eq. (3.2), we see that the $\alpha^{\prime}$ dependence cancels, as it must. The ultraviolet finite string amplitude, Eq. (3.2), has been replaced by a field theory amplitude which diverges in four space-time dimensions, because of the pole in the $\Gamma$ function in Eq. (3.11). Reverting to the customary conventions of dimensional regularization, we set $d=4-2 \epsilon$ and $g_{d}=g \mu^{\epsilon}$, where $g$ is dimensionless and $\mu$ an arbitrary scale. Putting everything together we find

$$
\begin{aligned}
A^{(1)}\left(p_{1}, p_{2}\right) & =-N \delta^{a_{1} a_{2}} \frac{g^{2}}{(4 \pi)^{2}}\left(\frac{4 \pi \mu^{2}}{-p_{1} \cdot p_{2}}\right)^{\epsilon} \varepsilon_{1} \cdot \varepsilon_{2} p_{1} \cdot p_{2} \\
& \times \Gamma(\epsilon) \frac{11-7 \epsilon}{3-2 \epsilon} B(1-\epsilon, 1-\epsilon) .
\end{aligned}
$$

Eq. (3.12) is exactly equal to the gluon vacuum polarization of Yang-Mills theory, computed with the background field method, in Feynman gauge, with dimensional regularization.

From Eq. (3.12) we see that the ultraviolet divergence at $d=4$ can be removed by a wave function renormalization, with a minimal subtraction $Z$ factor given by

$$
Z_{A}=1+N \frac{g^{2}}{(4 \pi)^{2}} \frac{11}{3} \frac{1}{\epsilon}
$$

While this result is what we expected, to make sure that our prescription is consistent we need to go on to compute the three and four-point renormalizations as well, and verify that gauge invariance is preserved. This we will do in the next two sections.

\section{The three-gluon amplitude}

The color-ordered amplitude for three transverse gluons at tree level is given by Eq. (2.21) with $M=3$, that is

$$
\begin{aligned}
A^{(0)}\left(p_{1}, p_{2}, p_{3}\right)= & -4 g_{d} \operatorname{Tr}\left(\lambda^{a_{1}} \lambda^{a_{2}} \lambda^{a_{3}}\right)\left(\varepsilon_{1} \cdot \varepsilon_{2} p_{2} \cdot \varepsilon_{3}\right. \\
& \left.+\varepsilon_{2} \cdot \varepsilon_{3} p_{3} \cdot \varepsilon_{1}+\varepsilon_{3} \cdot \varepsilon_{1} p_{1} \cdot \varepsilon_{2}+O\left(\alpha^{\prime}\right)\right)
\end{aligned}
$$

where $O\left(\alpha^{\prime}\right)$ stands for string corrections, proportional to $\alpha^{\prime}$, such as $\varepsilon_{1} \cdot p_{2} \varepsilon_{2} \cdot p_{3} \varepsilon_{3} \cdot p_{1}$. Since we are ultimately interested in the field theory limit, it is not necessary to 
write such terms explicitly. As is well known, when $\alpha^{\prime} \rightarrow 0, A^{(0)}\left(p_{1}, p_{2}, p_{3}\right)$ in Eq. (4.1) becomes the standard Yang-Mills color-ordered three-gluon amplitude, and the reader may easily verify the correctness of the normalization, taking into account our conventions.

The one-loop correction to Eq. (4.1) is given by

$$
\begin{aligned}
A^{(1)}\left(p_{1}, p_{2}, p_{3}\right) & =-N \operatorname{Tr}\left(\lambda^{a_{1}} \lambda^{a_{2}} \lambda^{a_{3}}\right) \frac{g_{d}^{3}}{(4 \pi)^{d / 2}}\left(2 \alpha^{\prime}\right)^{2-d / 2} \\
& \times \int_{0}^{\infty} \mathcal{D} \tau \int_{0}^{\tau} d \nu_{3} \int_{0}^{\nu_{3}} d \nu_{2} f_{3}\left(\nu_{2}, \nu_{3}, \tau\right)
\end{aligned}
$$

where

$$
\begin{aligned}
f_{3}\left(\nu_{2}, \nu_{3}, \tau\right) \equiv & \mathrm{e}^{2 \alpha^{\prime} p_{1} \cdot p_{2} G\left(\nu_{2}\right)} \mathrm{e}^{2 \alpha^{\prime} p_{2} \cdot p_{3} G\left(\nu_{32}\right)} \mathrm{e}^{2 \alpha^{\prime} p_{3} \cdot p_{1} G\left(\nu_{3}\right)} \\
\times & \left\{\left[-\varepsilon_{1} \cdot \varepsilon_{2} \partial_{2}^{2} G\left(\nu_{2}\right)\left(p_{1} \cdot \varepsilon_{3} \partial_{3} G\left(\nu_{3}\right)+p_{2} \cdot \varepsilon_{3} \partial_{3} G\left(\nu_{32}\right)\right)\right.\right. \\
& +\varepsilon_{2} \cdot \varepsilon_{3} \partial_{3}^{2} G\left(\nu_{32}\right)\left(p_{2} \cdot \varepsilon_{1} \partial_{2} G\left(\nu_{2}\right)+p_{3} \cdot \varepsilon_{1} \partial_{3} G\left(\nu_{3}\right)\right) \\
& \left.+\varepsilon_{1} \cdot \varepsilon_{3} \partial_{3}^{2} G\left(\nu_{3}\right)\left(p_{3} \cdot \varepsilon_{2} \partial_{3} G\left(\nu_{32}\right)-p_{1} \cdot \varepsilon_{2} \partial_{2} G\left(\nu_{2}\right)\right)\right] \\
& \left.+O\left(\alpha^{\prime}\right)\right\} .
\end{aligned}
$$

Our task is now to compare $A^{(1)}\left(p_{1}, p_{2}, p_{3}\right)$ with $A^{(0)}\left(p_{1}, p_{2}, p_{3}\right)$ in the limit $\alpha^{\prime} \rightarrow 0$, and thus derive the three-gluon vertex renormalization constant at one loop.

A careful analysis of Eq. (4.2) allows us to distinguish three different types of regions in the integration domain that contribute to the field theory limit, and to associate the three corresponding contributions to the three classes of Feynman diagrams depicted in Figs. 1 13 . To understand this, let us concentrate on two of the punctures, say $\nu_{2}$ and $\nu_{3}$, and follow the reasoning of Section 3 , which again leads us to identify the field theory limit of the amplitude (4.2) with the limit $\tau \rightarrow \infty$ in the integrand (4.3). There are now, however, three different ways of taking this limit that give finite contributions. First of all, one can let

$$
\tau \rightarrow \infty \quad, \quad \hat{\nu}_{3}-\hat{\nu}_{2} \equiv \hat{\nu}_{32}=O(1) \quad,
$$

which corresponds to shrinking the width of the annulus, while keeping the punctures widely separated. This region is strictly analogous to the only region that contributes to the two-point function, and can be discussed along the same lines of Section 3. We refer to it as a region of type $I$. As we shall see, this region will generate the contribution of one-particle irreducible diagrams with three propagators in the loop, and thus only three-point vertices, as shown in Fig. 1.

\footnotetext{
${ }^{3}$ Once again we omit the subscript $P$, as only the planar one-loop diagram contributes for $S U(N)$.
} 


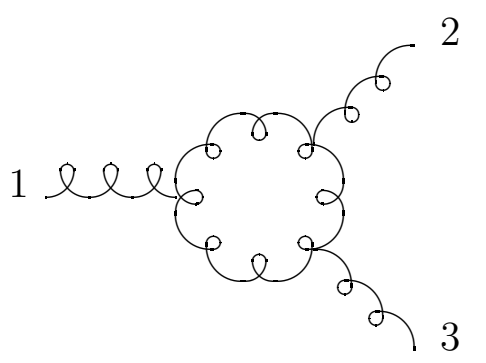

Figure 1: Representative diagram for the type $I$ region of the three-gluon amplitude.

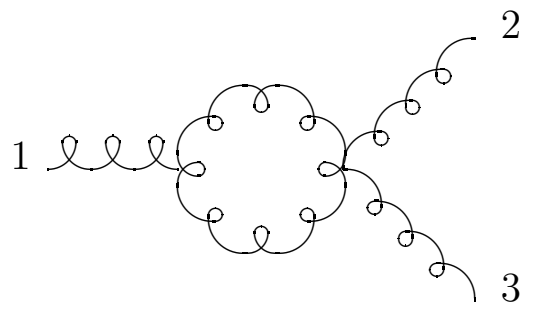

Figure 2: Representative diagram for the type $I I$ region $\hat{\nu}_{32}=O\left(\tau^{-1}\right)$ of the three-gluon amplitude.

The second relevant region is

$$
\tau \rightarrow \infty \quad, \quad \hat{\nu}_{32}=O\left(\tau^{-1}\right) .
$$

Here the $\nu$ coordinates are still widely separated, since $\nu_{3}-\nu_{2}=O(1)$, but the proper time of the corresponding propagator vanishes as $\tau \rightarrow \infty$, so that the two punctures come together in the field theory limit. This region, which we call of type $I I$, will generate the contribution of a diagram with a four-point vertex and only two propagators in the loop, as shown in Fig. 2. Notice that since the $\nu$ 's are still widely separated, the expansion given in Eq. (3.6) for the Green function $G\left(\nu_{32}\right)$ can still be applied, as we are still away from the singularity corresponding to $\nu_{2}=\nu_{3}$.

Finally, the third relevant region is the pinching region, defined by

$$
\tau \rightarrow \infty \quad, \quad \hat{\nu}_{32}=O\left(\tau^{-2}\right),
$$

so that in the limit $\tau \rightarrow \infty$ one approaches the logarithmic singularity of $G\left(\nu_{32}\right)$ as $\nu_{32} \rightarrow 0$. In this region, which we call of type $I I I$, the expansion in Eq. (3.6) is no 


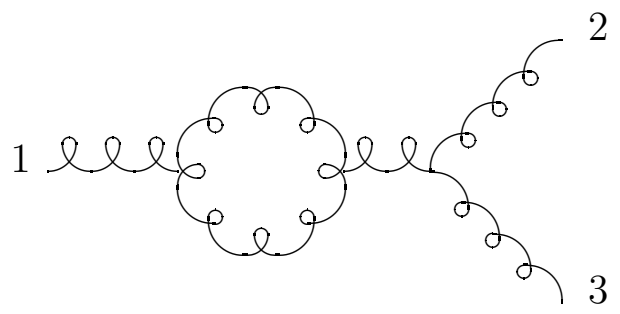

Figure 3: Representative diagram for the pinching region $\hat{\nu}_{32}=O\left(\tau^{-2}\right)$ of the three-gluon amplitude.

longer applicable, and one must use instead

$$
G(\nu) \sim \log (2 \nu)
$$

for the Green function and its derivatives. As shown already in Ref. [8], pinching two variables is equivalent to the introduction of a propagator attached to the loop, and thus generates an exchange diagram in the channel determined by the two pinched punctures. When such an exchange involves only gluons, the corresponding contribution to the amplitude in the limit $\alpha^{\prime} \rightarrow 0$ certainly corresponds to a one-particle reducible diagram, as shown in Fig. 3. However, there may be cases in which after pinching, the resulting exchange involves also the tachyon of the bosonic string. This leads to a divergence which we discard by hand, as we did for the divergence due to tachyons circulating in the loop. As we shall see in Section 5, there are circumstances in which the propagation of a tachyon in a pinched configuration can give a contribution that survives in the field theory limit, in the form of a contact interaction among four gluons. Such contributions are properly dealt with by assigning them to a type- $I I$ region, together with all other contributions corresponding to four-point vertices directly attached to the loop.

Since we are interested in minimal subtraction renormalization constants, in the following we will concentrate on the contributions that diverge as $d \rightarrow 4$. They are easy to isolate, as they can be produced only by an integral over the modular parameter $\tau$ containing the factor $\tau^{1-d / 2}$, that gives $\Gamma(2-d / 2)$ (lower powers of $\tau$ are suppressed by corresponding extra powers of $\left.\alpha^{\prime}\right)$. Since each $\nu$ integration generates a factor of $\tau$ through the change of variable $\nu \rightarrow \hat{\nu}$, we actually need the square bracket in Eq. (4.3) to generate a single negative power of $\tau$, in order to produce a divergent integral.

In order to display the relevant contributions, let's focus our attention on the term of Eq. (4.2) proportional to $\varepsilon_{1} \cdot \varepsilon_{2}$ which, up to the constant prefactors, is given by

$$
J\left(p_{1}, p_{2}, p_{3}\right)=\int_{0}^{\infty} d \tau \mathrm{e}^{2 \tau} \tau^{-d / 2} \prod_{n=1}^{\infty}\left(1-\mathrm{e}^{-2 n \tau}\right)^{2-d} \int_{0}^{\tau} d \nu_{3} \int_{0}^{\nu_{3}} d \nu_{2}
$$




$$
\begin{aligned}
& \times \mathrm{e}^{2 \alpha^{\prime} p_{1} \cdot p_{2} G\left(\nu_{2}\right)} \mathrm{e}^{2 \alpha^{\prime} p_{2} \cdot p_{3} G\left(\nu_{32}\right)} \mathrm{e}^{2 \alpha^{\prime} p_{3} \cdot p_{1} G\left(\nu_{3}\right)} \\
& \times\left[-\varepsilon_{1} \cdot \varepsilon_{2} \partial_{2}^{2} G\left(\nu_{2}\right)\left(p_{1} \cdot \varepsilon_{3} \partial_{3} G\left(\nu_{3}\right)+p_{2} \cdot \varepsilon_{3} \partial_{3} G\left(\nu_{32}\right)\right)\right]
\end{aligned}
$$

The other terms of $A^{(1)}\left(p_{1}, p_{2}, p_{3}\right)$ can be obtained by cyclic permutations from $J\left(p_{1}, p_{2}, p_{3}\right)$.

We start from the region of type $I$ defined by

$$
\tau \rightarrow \infty, \quad \hat{\nu}_{i+1, i}=O(1) \text { for all } 1 \leq i \leq 3
$$

where $\hat{\nu}_{43} \equiv 1-\hat{\nu}_{3}$. Here we can use the first term of the expansion of $\partial_{\nu}^{2} G(\nu)$, Eq. (3.9), in order to have the right power of $\tau$. Then we notice that no term in the expansion of $\partial_{\nu} G(\nu)$, Eq. (3.8), can exactly compensate the factor of $k^{-1}=\mathrm{e}^{2 \tau}$ in the measure, that signals the presence of the tachyon in the loop. Thus we are forced to pick up a factor of $k$ from the expansion of $\prod_{n}\left(1-k^{n}\right)^{2-d}$. Singling out these terms in the expansion of Eq. (4.8), we find that the total contribution to the ultraviolet divergence from region $I$ can be written as

$$
\begin{aligned}
\left.J\left(p_{1}, p_{2}, p_{3}\right)\right|_{I} & =-2(d-2) \varepsilon_{1} \cdot \varepsilon_{2} p_{2} \cdot \varepsilon_{3} \int_{0}^{\infty} d \tau \tau^{2-d / 2}\left(-\frac{2}{\tau}\right) \int_{0}^{1} d \hat{\nu}_{3} \int_{0}^{\hat{\nu}_{3}} d \hat{\nu}_{2} \\
& \times\left(\hat{\nu}_{3}-\hat{\nu}_{32}\right) \mathrm{e}^{2 \alpha^{\prime} p_{1} \cdot p_{2} G\left(\nu_{2}\right)} \mathrm{e}^{2 \alpha^{\prime} p_{2} \cdot p_{3} G\left(\nu_{32}\right)} \mathrm{e}^{2 \alpha^{\prime} p_{3} \cdot p_{1} G\left(\nu_{3}\right)}
\end{aligned}
$$

If we are only interested in the coefficient of the $1 / \epsilon$ pole, we can simply replace the exponentials with an infrared cutoff of the form $\exp \left(-2 \alpha^{\prime} m^{2} \tau\right)$. Then

$$
\left.J\left(p_{1}, p_{2}, p_{3}\right)\right|_{I}=\frac{4}{3} \varepsilon_{1} \cdot \varepsilon_{2} p_{2} \cdot \varepsilon_{3} \frac{1}{\epsilon}+O\left(\epsilon^{0}\right)
$$

We can now check that the region of moduli space just considered in fact corresponds to the diagrams in field theory with three propagators in the loop. Employing the usual Feynman rules of the background field method [21] one easily finds that these diagrams give in fact the same result as Eq. (4.11).

Let us now turn our attention to the regions of type $I I$. There are three such regions, all characterized by $\tau \rightarrow \infty$, while

$$
\hat{\nu}_{32}=O\left(\tau^{-1}\right)
$$

or

$$
1-\hat{\nu}_{3}=O\left(\tau^{-1}\right)
$$

or

$$
\hat{\nu}_{2}=O\left(\tau^{-1}\right)
$$

Region (4.12) corresponds to a vanishing proper time between the punctures $\nu_{2}$ and $\nu_{3}$ and can be associated with the diagram in Fig. 2. Similarly, if we keep in mind that the two points $\nu_{1}=0$ and $\tau$ are to be identified, we realize that the region 


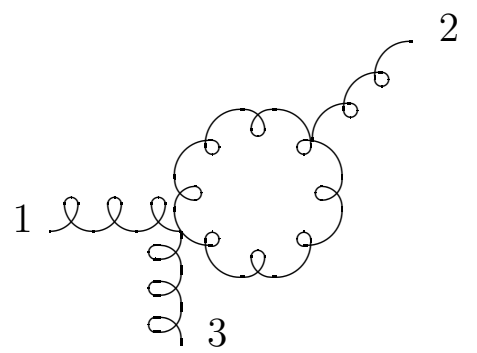

Figure 4: Representative diagram for the type $I I$ region $1-\hat{\nu}_{3}=O\left(\tau^{-1}\right)$ of the three-gluon amplitude.

in Eq. (4.13) corresponds to a vanishing proper time between $\nu_{3}$ and $\nu_{1}$, and thus it can be associated with the diagram represented in Fig. 4 . Finally, the third region, Eq. (4.14), corresponds to a vanishing proper time between $\nu_{1}$ and $\nu_{2}$ and can be associated with a diagram with two propagators and a four-point vertex between the gluons 1 and 2 .

In order to see how these regions contribute to $J\left(p_{1}, p_{2}, p_{3}\right)$, we use for $\partial_{\nu} G(\nu)$ and $\partial_{\nu}^{2} G(\nu)$ the terms of their expansions, Eqs. (3.8) and (3.9), that are of the form $k^{\hat{\nu}}$. They combine to give

$$
-8 \varepsilon_{1} \cdot \varepsilon_{2} p_{1} \cdot \varepsilon_{3}\left(k^{\left(1-\hat{\nu}_{32}\right)}-k^{\left(1+\hat{\nu}_{32}\right)}\right)+\ldots
$$

in region $(4.12)$, and

$$
8 \varepsilon_{1} \cdot \varepsilon_{2} p_{2} \cdot \varepsilon_{3}\left(k^{\hat{\nu}_{3}}-k^{\left(2-\hat{\nu}_{3}\right)}\right)+\ldots
$$

in region (4.13). For general values of $\hat{\nu}_{2}$ and $\hat{\nu}_{3}$ such terms, as well as all the others which we have not exhibited, fail to compensate the leading factor of $k^{-1}$ from the measure. However, precisely in the regions (4.12) and (4.13) the terms written in Eqs. (4.15) and (4.16) become proportional to $k$, and thus give a finite contribution. Once again, if we are only interested in the ultraviolet divergence we can replace the exponential of the Green functions by an infrared cutoff as above, whereupon the integral over the punctures becomes trivial. Indeed, the expression in Eq. (4.15) yields a factor of $(8 / \tau)\left(\varepsilon_{1} \cdot \varepsilon_{2} p_{1} \cdot \varepsilon_{3}\right)$ from region (4.12), while Eq. (4.16) produces a factor of $(-8 / \tau)\left(\varepsilon_{1} \cdot \varepsilon_{2} p_{2} \cdot \varepsilon_{3}\right)$ from region (4.13). On the other hand, one can check that region (4.14) does not give any contribution to the ultraviolet divergence of $J\left(p_{1}, p_{2}, p_{3}\right)$. In fact, in this case it is not possible to produce the right power of the modular parameter $\tau$ from the $\hat{\nu}$ integration.

Recalling that $p_{1} \cdot \varepsilon_{3}=-p_{2} \cdot \varepsilon_{3}$, the total contribution to $J\left(p_{1}, p_{2}, p_{3}\right)$ from regions of type $I I$ is then

$$
\left.J\left(p_{1}, p_{2}, p_{3}\right)\right|_{I I}=-16 \varepsilon_{1} \cdot \varepsilon_{2} p_{2} \cdot \varepsilon_{3} \frac{1}{\epsilon}+O\left(\epsilon^{0}\right)
$$


and one can verify that this is exactly the contribution from diagrams with a fourgluon vertex in the background field method, as conjectured.

Finally, we turn to the analysis of the pinching regions. In our case, there are three such regions, namely $\tau \rightarrow \infty$ with

$$
\hat{\nu}_{2}=O\left(\tau^{-2}\right)
$$

or

$$
\hat{\nu}_{32}=O\left(\tau^{-2}\right)
$$

or

$$
1-\hat{\nu}_{3}=O\left(\tau^{-2}\right)
$$

as dictated by cyclic symmetry and periodicity on the annulus. One can easily see that in all those regions at least one of the Green functions in Eq. (4.8) is singular.

Let us consider, for example, the first pinching, i.e. $\nu_{2} \rightarrow 0$. Since $\nu_{2}$ is localized in a neighbourhood of 0 , we can replace the integral $\int_{0}^{\nu_{3}} d \nu_{2}$ with an integral $\int_{0}^{\eta} d \nu_{2}$, where $\eta$ is an arbitrary small number. Further, we can use the approximation in Eq. (4.7) for the Green function $G\left(\nu_{2}\right)$ and its derivatives. After this is done, we can expand $G\left(\nu_{32}\right)$ in powers of $\nu_{2}$, which turns the amplitude $J\left(p_{1}, p_{2}, p_{3}\right)$ into an infinite series. The $n$-th term of this series is proportional to an integral of the form

$$
C_{n} \equiv \int_{0}^{\eta} d \nu_{2} \nu_{2}^{n-2+2 \alpha^{\prime} p_{1} \cdot p_{2}}
$$

with $n \geq 0$. After a suitable analytic continuation in the momenta to insure convergence, we get

$$
C_{n}=\frac{\eta^{n-1+2 \alpha^{\prime} p_{1} \cdot p_{2}}}{n-1+2 \alpha^{\prime} p_{1} \cdot p_{2}}
$$

Therefore, when the pinching $\nu_{2} \rightarrow 0$ is performed, the amplitude $J\left(p_{1}, p_{2}, p_{3}\right)$ becomes an infinite sum over all possible string states that are exchanged in the (12)-channel, $n=0$ corresponding to the tachyon, $n=1$ to the gluon and so on. In the case of the three-gluon amplitude, the exchange of a tachyon does not give any contribution: in fact, for $\nu_{2} \rightarrow 0$

$$
\begin{aligned}
\partial_{2}^{2} G & \left(\nu_{2}\right)\left(p_{1} \cdot \varepsilon_{3} \partial_{3} G\left(\nu_{3}\right)+p_{2} \cdot \varepsilon_{3} \partial_{3} G\left(\nu_{32}\right)\right) \\
& =-\frac{1}{\nu_{2}^{2}}\left(p_{1}+p_{2}\right) \cdot \varepsilon_{3} \partial_{3} G\left(\nu_{3}\right)+\frac{1}{\nu_{2}} p_{2} \cdot \varepsilon_{3} \partial_{3}^{2} G\left(\nu_{3}\right)+\ldots
\end{aligned}
$$

Using the trasversality of the external states, we see that the coefficient of the quadratic divergence $\frac{1}{\nu_{2}^{2}}$ is zero. Even if this tachyon contribution had not been vanishing, we would have discarded it, according to our prescription. On the other hand, the gluon term survives in the field theory limit, and actually contributes to the ultraviolet divergence, as expected: in fact, the single pole in $\nu_{2}$ generates, through the change of variables to $\hat{\nu}_{2}$, the negative power of $\tau$ needed for the integral 


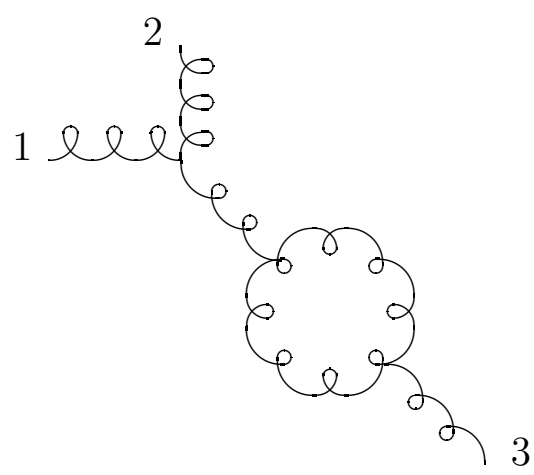

Figure 5: Representative diagram for the pinching region $\nu_{2} \rightarrow 0$ of the three-gluon amplitude.

to diverge. All other terms in the series, corresponding to $n \geq 2$, and to states whose mass becomes infinite as $\alpha^{\prime} \rightarrow 0$, vanish in the field theory limit.

Keeping this in mind, and collecting all relevant factors, we find that the contribution to $J\left(p_{1}, p_{2}, p_{3}\right)$ from the pinching $\nu_{2} \rightarrow 0$ is

$$
\left.J\left(p_{1}, p_{2}, p_{3}\right)\right|_{\nu_{2} \rightarrow 0}=\frac{\left(p_{1}+p_{2}\right) \cdot p_{3}}{p_{1} \cdot p_{2}} R\left[\left(p_{1}+p_{2}\right) \cdot p_{3}\right] \varepsilon_{1} \cdot \varepsilon_{2} p_{2} \cdot \varepsilon_{3}
$$

where $R$ is the integral defined in Eq. (3.5). Notice that Eq. (4.24) contains a ratio of momentum invariants which are vanishing on shell. The appearance of such ratios in string amplitudes, in the corners of moduli space corresponding to loops isolated on external legs, is a well-known fact, which for example motivated the work of Ref. [20]. As we already remarked in Ref. [15], this " $0 / 0$ " ambiguity is similar to the one that appears in the unrenormalized connected Green functions of a massless field theory, if the external legs are kept on the mass-shell and divergences are regularized with dimensional regularization. There, such ambiguity is removed by going off shell; similarly here, we formally continue the gluon momenta off the mass shell, following the same prescription we already adopted in the previous section for the two-gluon amplitude, i.e. we put off shell the momentum of the gluon attached to the loop, according to

$$
p_{3}^{2}=\left(p_{1}+p_{2}\right)^{2}=m^{2}
$$

In the present case, however, this prescription is not enough to remove the ambiguity in Eq. (4.24), and we must further decide whether and how to continue off shell also the other gluon momenta, $p_{1}$ and $p_{2}$. Here we rely on the assumption, substantiated by the results obtained so far, that string amplitudes lead to field theory amplitudes calculated with the background field method. As was shown in Ref. [22], to calculate amplitudes with the background field method it is necessary to treat one-particle 
irreducible and one-particle reducible diagrams quite differently. $S$-matrix elements are obtained by first calculating one-particle irreducible vertices to the desired order, and then gluing them together with propagators that can only be defined when the gauge for the background field has been fixed. As noticed also in Ref. [11], the gauge chosen for the background field is quite independent from the gauge that had been chosen for the quantum field, in our case the Feynman gauge. This leads us to interpret Eq. (4.24) as a one-loop, one-particle irreducible two-point function, whose momentum must be continued off shell according to Eq. (4.25), glued to a tree-level three-point vertex, for which no off-shell continuation is necessary. We thus keep $p_{1}^{2}=p_{2}^{2}=0$, which, using momentum conservation, implies

$$
\frac{\left(p_{1}+p_{2}\right) \cdot p_{3}}{p_{1} \cdot p_{2}}=-2
$$

Then, with this prescription, we find

$$
\begin{aligned}
\left.J\left(p_{1}, p_{2}, p_{3}\right)\right|_{\nu_{2} \rightarrow 0} & =-2 R\left(-m^{2}\right) \varepsilon_{1} \cdot \varepsilon_{2} p_{2} \cdot \varepsilon_{3} \\
& =\frac{44}{3} \varepsilon_{1} \cdot \varepsilon_{2} p_{2} \cdot \varepsilon_{3} \frac{1}{\epsilon}+O\left(\epsilon^{0}\right),
\end{aligned}
$$

where we have used Eq. (3.11) with $d=4-2 \epsilon$. One can easily check that this is exactly the contribution of one-particle reducible diagrams with the loop isolated on the third leg as shown in Fig. 5 .

The other two pinchings, Eq. (4.19) and Eq. (4.20), can be analyzed in a similar way with obvious changes of labels. One finds that they lead to the same result of Eq. (4.27) and can be associated with the diagrams represented in Fig. 3 and Fig. 6 with the loop isolated on the first and the second leg respectively. The total contribution to $J\left(p_{1}, p_{2}, p_{3}\right)$ from the type $I I I$ region is then

$$
\left.J\left(p_{1}, p_{2}, p_{3}\right)\right|_{I I I}=44 \varepsilon_{1} \cdot \varepsilon_{2} p_{2} \cdot \varepsilon_{3} \frac{1}{\epsilon}+O\left(\epsilon^{0}\right) \quad .
$$

As we have mentioned before, the other two terms of the amplitude (4.2) that are proportional to $\varepsilon_{2} \cdot \varepsilon_{3}$ and $\varepsilon_{3} \cdot \varepsilon_{1}$, can be simply obtained from $J\left(p_{1}, p_{2}, p_{3}\right)$ by cyclic permutations of indices. We can now write the field theory limit of the full one-loop amplitude $A^{(1)}\left(p_{1}, p_{2}, p_{3}\right)$ and extract from it the vertex renormalization constant. Reinstating all normalization factors, and summing the contributions from regions $I, I I$ and $I I I$, we find that the total divergence is

$$
\left.A^{(1)}\left(p_{1}, p_{2}, p_{3}\right)\right|_{\mathrm{div}}=2 N \frac{g^{2}}{(4 \pi)^{2}} \frac{11}{3} \frac{1}{\epsilon} A^{(0)}\left(p_{1}, p_{2}, p_{3}\right)
$$

Comparing with what we expect from field theory for a connected three-point amplitude, namely

$$
\left.A^{(1)}\left(p_{1}, p_{2}, p_{3}\right)\right|_{\mathrm{div}}=\left(Z_{3}^{-1} Z_{A}^{3}-1\right) A^{(0)}\left(p_{1}, p_{2}, p_{3}\right)
$$




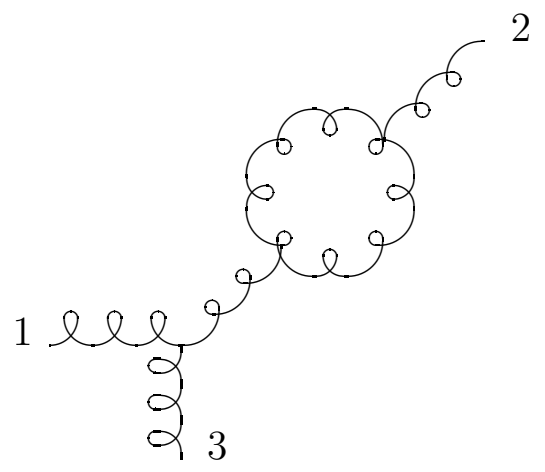

Figure 6: Representative diagram for the pinching region $\nu_{3} \rightarrow \tau$ of the three-gluon amplitude.

and using the result of Eq. (3.13), we are led to

$$
Z_{3}=1+N \frac{g^{2}}{(4 \pi)^{2}} \frac{11}{3} \frac{1}{\epsilon}=Z_{A}
$$

The Ward identity

$$
Z_{3}=Z_{A}
$$

is typical of the background field method. Thus our present result is a confirmation of what we have already found in Section of, and provides also a non-trivial consistency check on the whole procedure.

Further evidence of this fact is given by the following observation: since the contributions from regions $I$ and $I I$ are represented by the one-particle irreducible diagrams of Figs. 1, 2 and 4, while those of the pinching regions are given by the reducible diagrams of Figs. 3, 5 and 6, we are led to believe that the sum of the contributions from regions $I$ and $I I$ should be the proper three-point vertex and give directly the renormalization constant $Z_{3}$. This is exactly what happens. In fact,

$$
\begin{aligned}
\left.A^{(1)}\left(p_{1}, p_{2}, p_{3}\right)\right|_{1 \mathrm{PI}} & \left.\equiv A^{(1)}\left(p_{1}, p_{2}, p_{3}\right)\right|_{I}+\left.A^{(1)}\left(p_{1}, p_{2}, p_{3}\right)\right|_{I I} \\
& =-N \frac{g^{2}}{(4 \pi)^{2}} \frac{11}{3} \frac{1}{\epsilon} A^{(0)}\left(p_{1}, p_{2}, p_{3}\right)+O\left(\epsilon^{0}\right)
\end{aligned}
$$

which once again leads to the background field Ward identity (4.31).

In order to obtain these results, we could have adopted a slightly different procedure, integrating by parts the double derivatives of the Green functions as we did in Section 5. Doing that one arrives at the same result found by Bern, Kosower and Roland in Ref. [20] (they used a different string model, but that does not have any 
influence on the field theory limit). Integration by parts moves all contributions to the renormalization constants to the pinching regions, and thus spoils the identification of the different regions of moduli space with classes of Feynman diagrams, which is clearly displayed here. However, if one uses our prescription, Eq. (4.25) and Eq. (4.26), to remove the ambiguity associated with the pinching, Eq. (4.31) is again obtained.

As a final consistency check, we will verify the gauge invariance of our prescription, by calculating in the following section the renormalization of the four-point vertex, and showing that it is properly related to the renormalization of the gauge coupling.

\section{The four-gluon amplitude}

In order to obtain the renormalization constant $Z_{4}$ of the four-point vertex, we need to compare the four-gluon amplitude at one loop with the tree-level expression,

$$
\begin{aligned}
A^{(0)}\left(p_{1}, p_{2}, p_{3}, p_{4}\right) & =4 g_{d}^{2} \operatorname{Tr}\left(\lambda^{a_{1}} \lambda^{a_{2}} \lambda^{a_{3}} \lambda^{a_{4}}\right)\left(\varepsilon_{1} \cdot \varepsilon_{2} \varepsilon_{3} \cdot \varepsilon_{4} \frac{p_{1} \cdot p_{3}}{p_{1} \cdot p_{2}}\right. \\
& \left.+\varepsilon_{1} \cdot \varepsilon_{3} \varepsilon_{2} \cdot \varepsilon_{4}+\varepsilon_{1} \cdot \varepsilon_{4} \varepsilon_{2} \cdot \varepsilon_{3} \frac{p_{1} \cdot p_{3}}{p_{2} \cdot p_{3}}+\ldots\right)
\end{aligned}
$$

Here terms of the form $(\varepsilon \cdot \varepsilon)(\varepsilon \cdot p)^{2}$, as well as higher orders in $\alpha^{\prime}$, have not been written explicitly, since they will not play any role in our discussion. A more complete expression for $A^{(0)}\left(p_{1}, p_{2}, p_{3}, p_{4}\right)$ can be found in Eq. (A.6) of Appendix A.

The color-ordered planar amplitude with four gluons at one loop is given by Eq. (2.35) in the case $M=4$. Notice that in the open bosonic string the complete one-loop four-gluon amplitude receives a contribution also from the non-planar diagram, where two gluons are emitted at one boundary of the annulus and two at the other. However, the non-planar diagram does not contribute to the renormalization constant $Z_{4}$, so we need not consider it here.

To simplify the analysis, we will again focus on a single term of the full fourgluon amplitude. The simplest choice is the term proportional to $\varepsilon_{1} \cdot \varepsilon_{3} \varepsilon_{2} \cdot \varepsilon_{4}$ : in fact, for the chosen color ordering, at tree-level this term is generated only by the 1PI diagram given by the four-point vertex ${ }^{4}$. Thus, we will calculate the contributions from the three regions described in the previous section to the integral

$$
J\left(p_{1}, p_{2}, p_{3}, p_{4}\right)=\int_{0}^{\infty} d \tau \mathrm{e}^{2 \tau} \tau^{-d / 2} \prod_{n=1}^{\infty}\left(1-\mathrm{e}^{-2 n \tau}\right)^{2-d} \int_{0}^{\tau} d \nu_{4} \int_{0}^{\nu_{4}} d \nu_{3} \int_{0}^{\nu_{3}} d \nu_{2}
$$

\footnotetext{
${ }^{4}$ At the end of this section we will briefly comment on the term proportional to $\varepsilon_{1} \cdot \varepsilon_{2} \varepsilon_{3} \cdot \varepsilon_{4}$, which does not share this property.
} 


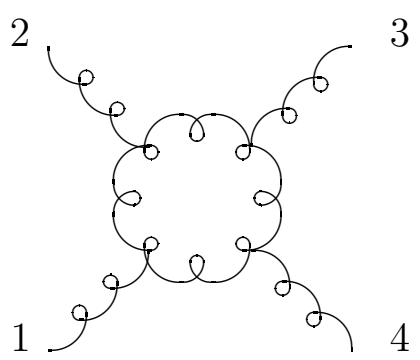

Figure 7: Representative diagram for the type $I$ region of the four-gluon amplitude.

$$
\begin{aligned}
& \times \quad \mathrm{e}^{2 \alpha^{\prime} p_{1} \cdot p_{2} G\left(\nu_{2}\right)} \mathrm{e}^{2 \alpha^{\prime} p_{1} \cdot p_{3} G\left(\nu_{3}\right)} \mathrm{e}^{2 \alpha^{\prime} p_{1} \cdot p_{4} G\left(\nu_{4}\right)} \\
& \times \quad \mathrm{e}^{2 \alpha^{\prime} p_{2} \cdot p_{3} G\left(\nu_{32}\right)} \mathrm{e}^{2 \alpha^{\prime} p_{2} \cdot p_{4} G\left(\nu_{42}\right)} \mathrm{e}^{2 \alpha^{\prime} p_{3} \cdot p_{4} G\left(\nu_{43}\right)} \\
& \times \quad \varepsilon_{1} \cdot \varepsilon_{3} \varepsilon_{2} \cdot \varepsilon_{4} \partial_{3}^{2} G\left(\nu_{3}\right) \partial_{4}^{2} G\left(\nu_{42}\right) .
\end{aligned}
$$

We start from region $I$, defined in this case by

$$
\tau \rightarrow \infty, \quad \hat{\nu}_{i+1, i}=O(1) \text { for all } 1 \leq i \leq 4
$$

where $\hat{\nu}_{54} \equiv 1-\hat{\nu}_{4}$. Since we are looking for those contributions that in the field theory limit diverge when $d \rightarrow 4$, again we need to isolate in the integrand of Eq. (5.2) the term proportional to $\tau^{1-d / 2}$. Then, to find the divergence, we can replace the exponentials of the Green functions by a simple infrared cutoff, as we did in Eq. (4.10). Substituting $\partial_{\nu}^{2} G(\nu)$ with the leading term of its expansion, Eq. (3.9), the contribution to $J\left(p_{1}, p_{2}, p_{3}, p_{4}\right)$ coming from region $I$ is

$$
\begin{aligned}
\left.J\left(p_{1}, p_{2}, p_{3}, p_{4}\right)\right|_{I} & =(d-2) \varepsilon_{1} \cdot \varepsilon_{3} \varepsilon_{2} \cdot \varepsilon_{4} \int_{0}^{\infty} d \tau \tau^{3-d / 2}\left(-\frac{2}{\tau}\right)^{2} \\
& \times \int_{0}^{1} d \hat{\nu}_{4} \int_{0}^{\hat{\nu}_{4}} d \hat{\nu}_{3} \int_{0}^{\hat{\nu}_{3}} d \hat{\nu}_{2} \mathrm{e}^{-2 \alpha^{\prime} m^{2} \tau} \\
& =\frac{4}{3} \varepsilon_{1} \cdot \varepsilon_{3} \varepsilon_{2} \cdot \varepsilon_{4} \frac{1}{\epsilon}+O\left(\epsilon^{0}\right) .
\end{aligned}
$$

We can check that this corresponds to the contribution of the field theory diagrams with four propagators in the loop, as depicted in Fig. 7.

In the previous section we learnt that factors of $1 / \tau$ are produced also by the type $I I$ regions. In the present case we have four such regions, namely

$$
\tau \rightarrow \infty \quad, \quad \hat{\nu}_{i+1, i}=O\left(\tau^{-1}\right) \quad, \quad 1 \leq i \leq 4
$$

To generate the desired factor of $1 / \tau$ we must recover in the integrand of Eq. (5.2) an expression similar to Eq. (4.15), and thus we must employ the last two terms of the expansion (3.9) for both of the factors $\partial_{\nu}^{2} G(\nu)$ present in Eq. (5.2). However, if 

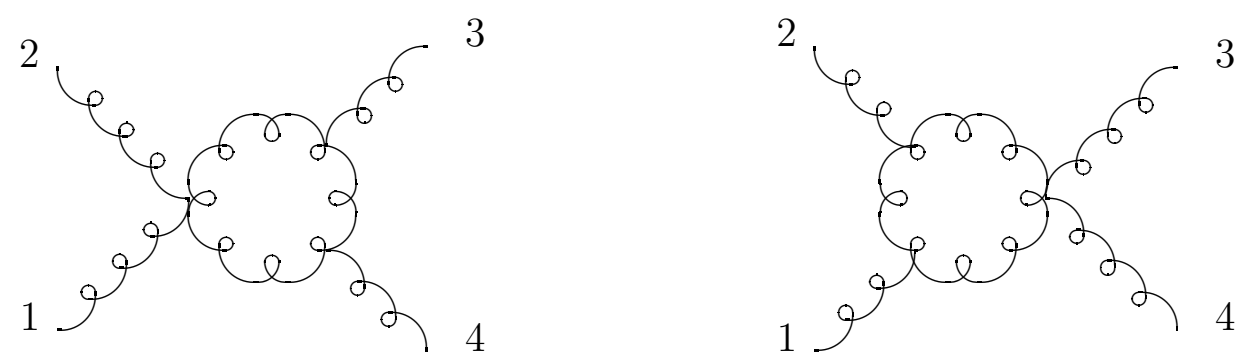

Figure 8: Representative diagrams that do not contribute to the divergence of the term proportional to $\varepsilon_{1} \cdot \varepsilon_{3} \varepsilon_{2} \cdot \varepsilon_{4}$ of the four-gluon amplitude. They instead contribute to the divergence of the term proportional to $\varepsilon_{1} \cdot \varepsilon_{2} \varepsilon_{3} \cdot \varepsilon_{4}$.

only one pair of $\hat{\nu}$ variables satisfies Eq. (5.5), while the others are widely separated, only one factor of $1 / \tau$ is produced by the integration, and the final result is not ultraviolet divergent in $d=4$. This means that diagrams with three propagators in the loop, like those depicted in Fig. 8, do not contribute to the coefficient of the $1 / \epsilon$ pole for the term $\varepsilon_{1} \cdot \varepsilon_{3} \varepsilon_{2} \cdot \varepsilon_{4}$. On the contrary, to produce a divergence we must consider two of the regions in Eq. (5.5) at the same time. We have the following two possibilities

$$
\tau \rightarrow \infty, \quad \hat{\nu}_{2}=O\left(\tau^{-1}\right) \quad \text { and } \quad \hat{\nu}_{43}=O\left(\tau^{-1}\right),
$$

or

$$
\tau \rightarrow \infty \quad, \quad \hat{\nu}_{32}=O\left(\tau^{-1}\right) \quad \text { and } \quad 1-\hat{\nu}_{4}=O\left(\tau^{-1}\right) .
$$

They correspond to the diagrams represented in Fig. 9 and Fig. 10 respectively, which have two propagators and two four-point vertices. The procedure is now almost identical to the one followed in Section 1 . After using Eq. (3.9), we find that the relevant terms of the form $k^{\hat{\nu}}$ combine to give

$$
16\left(k^{1+\hat{\nu}_{2}-\hat{\nu}_{43}}+k^{1+\hat{\nu}_{43}-\hat{\nu}_{2}}\right)+\ldots
$$

in region (5.6), and

$$
16\left(k^{\hat{\nu}_{32}+\hat{\nu}_{4}}+k^{2-\hat{\nu}_{4}-\hat{\nu}_{32}}\right)+\ldots
$$

in region (5.7). Computing the $\hat{\nu}$ integrals and adding the two contributions, we find

$$
\left.J\left(p_{1}, p_{2}, p_{3}, p_{4}\right)\right|_{I I}=-16 \varepsilon_{1} \cdot \varepsilon_{3} \varepsilon_{2} \cdot \varepsilon_{4} \frac{1}{\epsilon}+O\left(\epsilon^{0}\right) .
$$

Finally, we turn our attention to one-particle reducible contributions originating from the pinching regions. By analyzing the structure of the integrand of Eq. (5.2), one realizes that pinching singularities contribute to the ultraviolet divergence of the term we are examining only when three consecutive punctures are pinched together, 


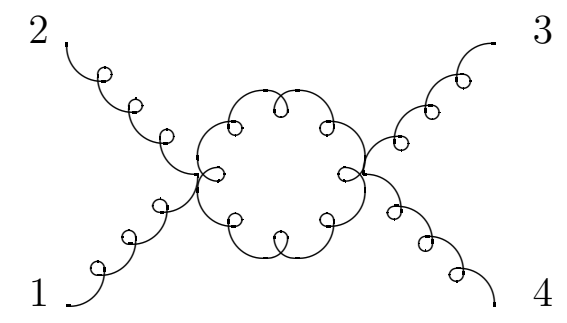

Figure 9: Representative diagram for the region $\hat{\nu}_{2}=O\left(\tau^{-1}\right)$ and $\hat{\nu}_{43}=O\left(\tau^{-1}\right)$ of the four-gluon amplitude.

that is when $\nu_{2} \rightarrow \nu_{3} \rightarrow \nu_{4}$, or $\nu_{3} \rightarrow \nu_{4} \rightarrow \tau$, or $\nu_{3} \rightarrow \nu_{2} \rightarrow 0$, or $\nu_{2} \rightarrow 0$ with $\nu_{4} \rightarrow \tau$. Each of these four regions gives the same contribution, that we expect to be simply proportional to the two-gluon amplitude, since the loop is isolated on an external leg, as shown in Fig. 11.

Let us concentrate on the pinching $\nu_{2} \rightarrow \nu_{3} \rightarrow \nu_{4}$, which isolates the loop on the first leg. In this case Eq. (5.2) becomes

$$
\begin{aligned}
\left.J\left(p_{1}, p_{2}, p_{3}, p_{4}\right)\right|_{\nu_{2} \rightarrow \nu_{3} \rightarrow \nu_{4}} & =-\varepsilon_{1} \cdot \varepsilon_{3} \varepsilon_{2} \cdot \varepsilon_{4} \int_{0}^{\infty} d \tau \mathrm{e}^{2 \tau} \tau^{-d / 2} \\
& \times \prod_{n=1}^{\infty}\left(1-\mathrm{e}^{-2 n \tau}\right)^{2-d} \int_{0}^{\tau} d \nu_{4} \int_{\nu_{4}-\eta}^{\nu_{4}} d \nu_{3} \\
& \times \int_{\nu_{3}-\eta}^{\nu_{3}} d \nu_{2} \mathrm{e}^{2 \alpha^{\prime}\left(p_{2}+p_{3}+p_{4}\right) \cdot p_{1} G\left(\nu_{4}\right)} \partial_{4}^{2} G\left(\nu_{4}\right) \\
& \times\left(\nu_{43}\right)^{2 \alpha^{\prime} p_{3} \cdot p_{4}}\left(\nu_{32}\right)^{2 \alpha^{\prime} p_{2} \cdot p_{3}}\left(\nu_{42}\right)^{-2+2 \alpha^{\prime} p_{2} \cdot p_{4}}
\end{aligned}
$$

where $\eta$ is an arbitrary small number. By means of the change of variables

$$
\nu_{3} \rightarrow x=\nu_{43} \quad, \quad \nu_{2} \rightarrow y=\frac{\nu_{32}}{\nu_{43}}
$$

the integrals over $\nu_{2}$ and $\nu_{3}$ decouple and become respectively

$$
\int_{0}^{\infty} d y(1+y)^{-2+2 \alpha^{\prime} p_{2} \cdot p_{4}} y^{2 \alpha^{\prime} p_{2} \cdot p_{3}}=1+O\left(\alpha^{\prime}\right)
$$

and

$$
\int_{0}^{\eta} d x x^{-1+2 \alpha^{\prime}\left(p_{2} \cdot p_{3}+p_{3} \cdot p_{4}+p_{2} \cdot p_{4}\right)} .
$$

Notice that this last integral is of the form of Eq. (4.21) with $n=1$. Then, for $\alpha^{\prime} \rightarrow 0$ we get

$$
\begin{aligned}
\left.J\left(p_{1}, p_{2}, p_{3}, p_{4}\right)\right|_{\nu_{2} \rightarrow \nu_{3} \rightarrow \nu_{4}} & =\varepsilon_{1} \cdot \varepsilon_{3} \varepsilon_{2} \cdot \varepsilon_{4} \frac{\left(p_{2}+p_{3}+p_{4}\right) \cdot p_{1}}{p_{2} \cdot p_{3}+p_{3} \cdot p_{4}+p_{2} \cdot p_{4}} \\
& \times R\left(\left(p_{2}+p_{3}+p_{4}\right) \cdot p_{1}\right) .
\end{aligned}
$$




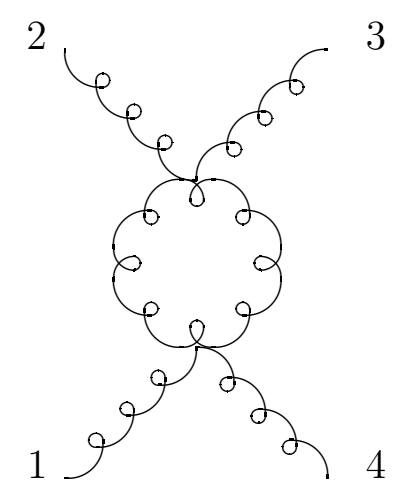

Figure 10: Representative diagram for the region $\hat{\nu}_{32}=O\left(\tau^{-1}\right)$ and $1-\hat{\nu}_{4}=O\left(\tau^{-1}\right)$ of the four-gluon amplitude.

As expected, this last equation contains a ratio of invariants that becomes indeterminate when the external states are on shell. We solve the ambiguity with the same prescription used in the previous section, i.e. we put off shell only the momentum of the gluon attached to the loop and keep the others on shell, according to

$$
p_{1}^{2}=m^{2} \quad, \quad p_{2}^{2}=p_{3}^{2}=p_{4}^{2}=0 \quad .
$$

Using momentum conservation and Eq. (5.16), we easily see that

$$
\frac{\left(p_{2}+p_{3}+p_{4}\right) \cdot p_{1}}{p_{2} \cdot p_{3}+p_{3} \cdot p_{4}+p_{2} \cdot p_{4}}=-2 \text {. }
$$

Hence

$$
\begin{aligned}
\left.J\left(p_{1}, p_{2}, p_{3}, p_{4}\right)\right|_{\nu_{2} \rightarrow \nu_{3} \rightarrow \nu_{4}} & =-2 R\left(-m^{2}\right) \varepsilon_{1} \cdot \varepsilon_{3} \varepsilon_{2} \cdot \varepsilon_{4} \\
& =\frac{44}{3} \varepsilon_{1} \cdot \varepsilon_{3} \varepsilon_{2} \cdot \varepsilon_{4} \frac{1}{\epsilon}+O\left(\epsilon^{0}\right),
\end{aligned}
$$

where again we have used Eq. (3.11) for $d=4-2 \epsilon$. The other three pinchings can be analyzed in a similar way and lead to the same result of Eq. (5.18). Therefore, the total contribution to $J\left(p_{1}, p_{2}, p_{3}, p_{4}\right)$ from region $I I I$ is

$$
\left.J\left(p_{1}, p_{2}, p_{3}, p_{4}\right)\right|_{I I I}=4 \frac{44}{3} \varepsilon_{1} \cdot \varepsilon_{3} \varepsilon_{2} \cdot \varepsilon_{4} \frac{1}{\epsilon}+O\left(\epsilon^{0}\right)
$$

Collecting all the results derived so far, and reinstating all normalization factors, we find that

$$
\left.A^{(1)}[13,24]\right|_{\mathrm{div}}=3 N \frac{g^{2}}{(4 \pi)^{2}} \frac{11}{3} \frac{1}{\epsilon} A^{(0)}[13,24],
$$

where the symbols $A^{(1)}[13,24]$ and $A^{(0)}[13,24]$ denote the terms proportional to $\varepsilon_{1} \cdot \varepsilon_{3} \varepsilon_{2} \cdot \varepsilon_{4}$ in the four-gluon amplitude at one loop and tree level respectively. 


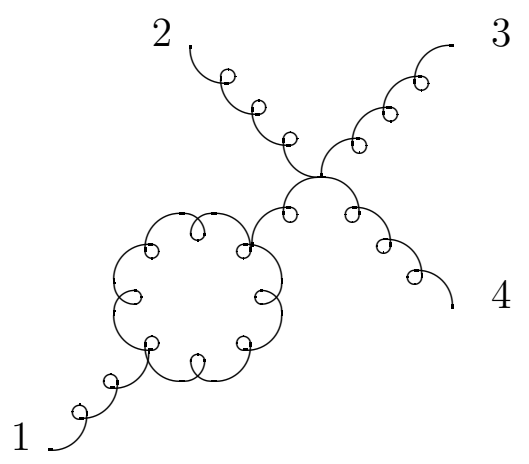

Figure 11: Representative diagram for the pinching $\nu_{2} \rightarrow \nu_{3} \rightarrow \nu_{4}$ in the four-gluon amplitude. The other three double pinchings, $\nu_{3} \rightarrow \nu_{4} \rightarrow \tau, \nu_{2} \rightarrow 0$ with $\nu_{4} \rightarrow \tau$, and $\nu_{3} \rightarrow \nu_{2} \rightarrow 0$, give rise to similar diagrams with the loop isolated respectively on the second, the third and the fourth external gluon.

Comparing with what we expect from field theory for a truncated, connected fourpoint Green function, we obtain

$$
Z_{4}=1+N \frac{g^{2}}{(4 \pi)^{2}} \frac{11}{3} \frac{1}{\epsilon}
$$

Thus, we are led to the background field Ward identities

$$
Z_{A}=Z_{3}=Z_{4}
$$

as desired.

This result is further confirmed by the fact that the contributions from the type $I$ and type $I I$ regions, associated with one-particle irreducible diagrams, properly add. Indeed,

$$
\begin{aligned}
\left.A^{(1)}[13,24]\right|_{1 \mathrm{PI}} & \left.\equiv A^{(1)}[13,24]\right|_{I}+\left.A^{(1)}[13,24]\right|_{I I} \\
& =-N \frac{g^{2}}{(4 \pi)^{2}} \frac{11}{3} \frac{1}{\epsilon} A^{(0)}[13,24]+O\left(\epsilon^{0}\right),
\end{aligned}
$$

which leads directly to Eq. (5.21).

We conclude with a brief analysis of the term proportional to $\varepsilon_{1} \cdot \varepsilon_{2} \varepsilon_{3} \cdot \varepsilon_{4}$ in the four-gluon amplitude, and show that our results, Eq. (5.21) and Eq. (5.22), still hold in this case. Let us then consider the integral

$$
\begin{aligned}
\tilde{J}\left(p_{1}, p_{2}, p_{3}, p_{4}\right) & =\int_{0}^{\infty} d \tau \mathrm{e}^{2 \tau} \tau^{-d / 2} \prod_{n=1}^{\infty}\left(1-\mathrm{e}^{-2 n \tau}\right)^{2-d} \int_{0}^{\tau} d \nu_{4} \int_{0}^{\nu_{4}} d \nu_{3} \int_{0}^{\nu_{3}} d \nu_{2} \\
& \times \mathrm{e}^{2 \alpha^{\prime} p_{1} \cdot p_{2} G\left(\nu_{2}\right)} \mathrm{e}^{2 \alpha^{\prime} p_{1} \cdot p_{3} G\left(\nu_{3}\right)} \mathrm{e}^{2 \alpha^{\prime} p_{1} \cdot p_{4} G\left(\nu_{4}\right)} \\
& \times \mathrm{e}^{2 \alpha^{\prime} p_{2} \cdot p_{3} G\left(\nu_{32}\right)} \mathrm{e}^{2 \alpha^{\prime} p_{2} \cdot p_{4} G\left(\nu_{42}\right)} \mathrm{e}^{2 \alpha^{\prime} p_{3} \cdot p_{4} G\left(\nu_{43}\right)} \\
& \times \varepsilon_{1} \cdot \varepsilon_{2} \varepsilon_{3} \cdot \varepsilon_{4} \partial_{2}^{2} G\left(\nu_{2}\right) \partial_{4}^{2} G\left(\nu_{43}\right)
\end{aligned}
$$


and let us compute its field theory limit. The contribution from the type $I$ region, Eq. (5.3), is as before, namely

$$
\left.\tilde{J}\left(p_{1}, p_{2}, p_{3}, p_{4}\right)\right|_{I}=\frac{4}{3} \varepsilon_{1} \cdot \varepsilon_{2} \varepsilon_{3} \cdot \varepsilon_{4} \frac{1}{\epsilon}+O\left(\epsilon^{0}\right)
$$

The contribution from the type $I I$ region is, however, different from Eq. (5.10). This can be traced to the fact that in this case there are contributions from regions of type $I I$ in which the two $\hat{\nu}$ variables that are taken to be close to each other are precisely the ones that appear in the argument of one of the Green functions whose double derivative is taken in Eq. (5.24). As a consequence, there will now be non-vanishing contributions also when a single pair of $\hat{\nu}$ variables approach each other. Let us, for instance, consider the region

$$
\tau \rightarrow \infty \quad, \quad \hat{\nu}_{2}=O\left(\tau^{-1}\right) \quad .
$$

with all other variables widely separated. It corresponds to the diagram depicted on the left in Fig. 8. Using Eq. (3.9), we see that in this region the relevant terms of $\partial_{2}^{2} G\left(\nu_{2}\right) \partial_{4}^{2} G\left(\nu_{43}\right)$ combine to give

$$
\frac{8}{\tau}\left(\sum_{n=1}^{\infty} n \mathrm{e}^{-2 n \tau \hat{\nu}_{2}}+\mathrm{e}^{-2 \tau\left(1-\hat{\nu}_{2}\right)}+\mathrm{e}^{-2 \tau\left(1+\hat{\nu}_{2}\right)}\right)+\ldots .
$$

Now however, in contrast to all previous cases, the entire series in $n$, and not just its first term, must be taken into account: in fact, in region (5.26) all terms of the form $\mathrm{e}^{-2 n \tau \hat{\nu}_{2}}$ are on equal footing for any $n$ since $\tau \hat{\nu}_{2}$ is finite. Computing the integrals, and extracting the right power of $\tau$ that is necessary to produce the divergence in $d=4-2 \epsilon$, we find that

$$
\begin{aligned}
\left.\tilde{J}\left(p_{1}, p_{2}, p_{3}, p_{4}\right)\right|_{\hat{\nu}_{2}=O\left(\tau^{-1}\right)} & =4\left(\sum_{n=1}^{\infty} 1\right) \varepsilon_{1} \cdot \varepsilon_{2} \varepsilon_{3} \cdot \varepsilon_{4} \frac{1}{\epsilon}+O\left(\epsilon^{0}\right) \\
& =-2 \varepsilon_{1} \cdot \varepsilon_{2} \varepsilon_{3} \cdot \varepsilon_{4} \frac{1}{\epsilon}+O\left(\epsilon^{0}\right)
\end{aligned}
$$

where we have regularized the divergent sum $\sum_{n=1}^{\infty} 1$ by means of the formula

$$
\sum_{n=1}^{\infty} 1=\lim _{s \rightarrow 0} \sum_{n=1}^{\infty} n^{-s}=\zeta(0)=-\frac{1}{2} .
$$

It should be apparent that the reason for this divergence is the singularity of the Green function as $\nu_{2} \rightarrow 0$, which did not cause problems when only the first term in the series in Eq. (3.7) was used, but cannot be avoided when the whole series is included. One may thus wonder whether we are not double counting some terms,

\footnotetext{
${ }^{5}$ This $\zeta$-function regularization will be used also in Section 6, following [2].
} 
which might as well be included in the pinching region. This does not happen, and the reason is that the residual contribution from the pinching region, which is included in Eq. (5.28), can be traced to the exchange of a tachyon in the pinched channel, that is explicitly discarded when we study region $I I I$. In fact, if one uses Eq. (4.22) for $n=0$ to isolate the tachyon contribution in the relevant channel, the result is divergent in the field theory limit. This divergence can be regularized by taking the residue of the tachyon pole, which corresponds to a contact interaction with a four-gluon vertex and no propagating tachyon. This calculation leads precisely to a contribution matching Eq. (5.28). By including this contact interaction in region $I I$ we are correctly identifying it as a one-particle irreducible contribution.

A similar reasoning leads to identify an identical contribution from the region

$$
\tau \rightarrow \infty \quad, \quad \hat{\nu}_{43}=O\left(\tau^{-1}\right),
$$

which corresponds to the diagram depicted on the right of Fig. 8.

Finally, we must consider the contribution from regions (5.6) and (5.7), associated with the diagrams in Fig. 9 and Fig. 10 respectively. The relevant terms of $\partial_{2}^{2} G\left(\nu_{2}\right) \partial_{4}^{2} G\left(\nu_{43}\right)$ combine to give

$$
\begin{aligned}
& 16\left(\sum_{n=1}^{\infty} n \mathrm{e}^{-2 n \tau \hat{\nu}_{2}}+\mathrm{e}^{-2 \tau\left(1-\hat{\nu}_{2}\right)}+\mathrm{e}^{-2 \tau\left(1+\hat{\nu}_{2}\right)}\right) \\
& \times\left(\sum_{m=1}^{\infty} m \mathrm{e}^{-2 m \tau \hat{\nu}_{43}}+\mathrm{e}^{-2 \tau\left(1-\hat{\nu}_{43}\right)}+\mathrm{e}^{-2 \tau\left(1+\hat{\nu}_{43}\right)}\right)+\ldots,
\end{aligned}
$$

in region (5.6), while in region (5.7) they give

$$
16\left(\mathrm{e}^{-2 \tau\left(\hat{\nu}_{4}-\hat{\nu}_{32}\right)}+\mathrm{e}^{-2 \tau\left(2-\hat{\nu}_{4}+\hat{\nu}_{32}\right)}\right)+\ldots \quad .
$$

Computing the integrals we find that, after using twice the $\zeta$-function regularization, Eq. (5.31) yields

$$
2 \varepsilon_{1} \cdot \varepsilon_{2} \varepsilon_{3} \cdot \varepsilon_{4} \frac{1}{\epsilon}+O\left(\epsilon^{0}\right)
$$

while Eq. (5.32) yields

$$
8 \varepsilon_{1} \cdot \varepsilon_{2} \varepsilon_{3} \cdot \varepsilon_{4} \frac{1}{\epsilon}+O\left(\epsilon^{0}\right)
$$

Adding all contributions of the type $I I$ regions, we see that

$$
\left.\tilde{J}\left(p_{1}, p_{2}, p_{3}, p_{4}\right)\right|_{I I}=6 \varepsilon_{1} \cdot \varepsilon_{2} \varepsilon_{3} \cdot \varepsilon_{4} \frac{1}{\epsilon}+O\left(\epsilon^{0}\right) \quad .
$$

As we have discussed above, the regions of type $I$ and $I I$ are associated with diagrams that are 1PI. Then, adding the contributions of these regions, Eq. (5.25) and Eq. (5.35), and reinstating all normalization factors, we get, in obvious notations,

$$
\begin{aligned}
\left.A^{(1)}[12,34]\right|_{1 \mathrm{PI}} & \left.\equiv A^{(1)}[12,34]\right|_{I}+\left.A^{(1)}[12,34]\right|_{I I} \\
& =-\left.N \frac{g^{2}}{(4 \pi)^{2}} \frac{11}{3} \frac{1}{\epsilon} A^{(0)}[12,34]\right|_{1 \mathrm{PI}}+O\left(\epsilon^{0}\right),
\end{aligned}
$$


where $\left.A^{(0)}[12,34]\right|_{1 \mathrm{PI}}$ stands for the color-ordered tree-level 1PI term proportional to $\varepsilon_{1} \cdot \varepsilon_{2} \varepsilon_{3} \cdot \varepsilon_{4}$ in the Feynman gauge, that is

$$
\left.A^{(0)}[12,34]\right|_{1 \mathrm{PI}}=-2 g_{d}^{2} \operatorname{Tr}\left(\lambda^{a_{1}} \lambda^{a_{2}} \lambda^{a_{3}} \lambda^{a_{4}}\right) \varepsilon_{1} \cdot \varepsilon_{2} \varepsilon_{3} \cdot \varepsilon_{4}
$$

Clearly, Eq. (5.36) leads to the correct value of $Z_{4}$, Eq. (5.21).

Finally, the pinching contributions to $\tilde{J}\left(p_{1}, p_{2}, p_{3}, p_{4}\right)$ due to gluon exchanges can be easily computed along the lines discussed above. If we also add them, we get

$$
\left.A^{(1)}[12,34]\right|_{\mathrm{div}}=3 N \frac{g^{2}}{(4 \pi)^{2}} \frac{11}{3} \frac{1}{\epsilon} A^{(0)}[12,34],
$$

as expected.

\section{A direct computation of proper vertices}

From the detailed analysis of the previous sections we learnt that it is possible, and indeed desirable, if one wishes to calculate renormalization constants, to isolate from the amplitude one-particle irreducible diagrams that contribute to the effective action. In this section we will show how this can be done directly at the level of the string amplitude, following the ideas of Metsaev and Tseytlin [2]. The starting point of this approach is the introduction of an alternative expression for the bosonic Green function, Eq. (2.41), which is suitable for a regularization of pinching singularities. As we shall see, the $\zeta$-function regularization used in this case is precisely the one introduced in Eq. (5.29), and thus it handles correctly also the residual contributions from the propagation of the tachyon in the pinched channel, assigning them to one-particle irreducible diagrams. The most interesting feature of this approach is that, at least at one loop, it allows to integrate exactly over the punctures, before the field theory limit is taken. This is possible because, having regularized the pinching singularity, there is no integration region that generates negative powers of $\alpha^{\prime}$ in the field theory limit. One can then focus on terms proportional to $\left(\alpha^{\prime}\right)^{2-d / 2}$, and for those terms one is allowed to replace the exponentials of the Green function by a simple infrared cutoff, of the form $\exp \left(-2 \alpha^{\prime} m^{2} \tau\right)$, as we learnt in the previous sections. The simplified integral over the punctures can then be performed exactly, using the Green function given by Eq. (2.41). Using these techniques we will be able to express the renormalizations of the various divergent amplitudes in terms of a single string integral, $Z(d)$, which in the field theory limit reproduces the wave function renormalization $Z_{A}$.

Our starting point is Eq. (2.35), with the choice of projective transformations given in Eq. (2.38). We rewrite it as

$$
A_{P}^{(1)}\left(p_{1}, \ldots, p_{M}\right)=N \operatorname{Tr}\left(\lambda^{a_{1}} \cdots \lambda^{a_{M}}\right) \frac{g_{d}^{M}}{(4 \pi)^{d / 2}}\left(2 \alpha^{\prime}\right)^{2-d / 2}
$$




$$
\times \quad(-1)^{M} \int_{0}^{\infty} \mathcal{D} \tau I_{M}^{(1)}(\tau)
$$

where

$$
\begin{aligned}
I_{M}^{(1)}(\tau) & =\left(2 \alpha^{\prime}\right)^{M / 2-2} \int_{0}^{\tau} d \nu_{M} \int_{0}^{\nu_{M}} d \nu_{M-1} \cdots \int_{0}^{\nu_{3}} d \nu_{2}\left\{\prod_{i<j}\left[\exp \left(G\left(\nu_{j i}\right)\right)\right]^{2 \alpha^{\prime} p_{i} \cdot p_{j}}\right. \\
& \left.\times \exp \left[\sum_{i \neq j}\left(\sqrt{2 \alpha^{\prime}} p_{j} \cdot \varepsilon_{i} \partial_{i} G\left(\nu_{j i}\right)+\frac{1}{2} \varepsilon_{i} \cdot \varepsilon_{j} \partial_{i} \partial_{j} G\left(\nu_{j i}\right)\right)\right]\right\}_{\mathrm{m} . l .}
\end{aligned}
$$

For $M=2$, after a partial integration with vanishing surface term, we get

$$
I_{2}^{(1)}(\tau)=\varepsilon_{1} \cdot \varepsilon_{2} p_{1} \cdot p_{2} \int_{0}^{\tau} d \nu\left(\partial_{\nu} G(\nu)\right)^{2} \mathrm{e}^{2 \alpha^{\prime} p_{1} \cdot p_{2} G(\nu)} .
$$

As announced, since the overall power of $\alpha^{\prime}$ is already appropriate, we can now neglect the exponential, and replace it with an infrared cutoff to isolate ultraviolet divergences. Using the expression in Eq. (2.41) for the Green function, we can easily perform exactly the integral over the puncture, by means of the identity

$$
\int_{0}^{1} d x \sin (2 \pi n x) \sin (2 \pi m x)=\frac{1}{2} \delta_{n m}
$$

We get

$$
I_{2}^{(1)}(\tau)=\frac{2 \pi^{2}}{\tau} \varepsilon_{1} \cdot \varepsilon_{2} p_{1} \cdot p_{2} \sum_{n=1}^{\infty}\left(\frac{1+q^{2 n}}{1-q^{2 n}}\right)^{2},
$$

which allows one to write

$$
\begin{aligned}
\left.A^{(1)}\left(p_{1}, p_{2}\right)\right|_{\mathrm{div}} & =\frac{N}{2} \operatorname{Tr}\left(\lambda^{a_{1}} \lambda^{a_{2}}\right) \frac{g_{d}^{2}}{(4 \pi)^{d / 2}}\left(2 \alpha^{\prime}\right)^{2-d / 2} \varepsilon_{1} \cdot \varepsilon_{2} p_{1} \cdot p_{2} Z(d) \\
& =\frac{N}{4} \frac{g_{d}^{2}}{(4 \pi)^{d / 2}}\left(2 \alpha^{\prime}\right)^{2-d / 2} Z(d) A^{(0)}\left(p_{1}, p_{2}\right)
\end{aligned}
$$

Here

$$
Z(d) \equiv(2 \pi)^{2} \int_{0}^{\infty} \frac{\mathcal{D} \tau}{\tau} \sum_{m=1}^{\infty}\left(\frac{1+q^{2 m}}{1-q^{2 m}}\right)^{2}
$$

is the string integral that generates the renormalization constants as $\alpha^{\prime} \rightarrow 0$, and, with our normalizations

$$
A^{(0)}\left(p_{1}, p_{2}\right)=2 \operatorname{Tr}\left(\lambda^{a_{1}} \lambda^{a_{2}}\right) \varepsilon_{1} \cdot \varepsilon_{2} p_{1} \cdot p_{2}
$$

Since the integral $Z(d)$ will reappear in the analysis of the three and four-point amplitudes, we postpone its evaluation and go on to perform the integral over the punctures for the other amplitudes. 
With three gluons we get

$$
\begin{aligned}
I_{3}^{(1)}(\tau) & =\int_{0}^{\tau} d \nu_{3} \int_{0}^{\nu_{3}} d \nu_{2}\left\{-\varepsilon_{1} \cdot \varepsilon_{2} \partial_{2}^{2} G\left(\nu_{2}\right)\right. \\
& \left.\times\left[p_{1} \cdot \varepsilon_{3} \partial_{3} G\left(\nu_{3}\right)+p_{2} \cdot \varepsilon_{3} \partial_{3} G\left(\nu_{32}\right)\right]+\ldots\right\}
\end{aligned}
$$

where terms needed for cyclic symmetry and terms of order $\alpha^{\prime}$ are not written explicitly, and we discarded the exponentials of the Green functions, that are again irrelevant for ultraviolet divergences. Notice that for the three-point function the overall power of $\alpha^{\prime}$ is correct provided we do not perform the partial integration of double derivatives, as was done for the two-point amplitude. As remarked at the end of Section 1 , partial integration of the three-gluon integrand reshuffles the contributions of different diagrams in the field theory limit, so that it is no longer possible to identify unambiguously the one-particle irreducible ones.

The integrals over $\nu_{2}$ and $\nu_{3}$ can now be done, using as before Eq. (2.41) for the Green function. It is easier to start by performing the integral over $\nu_{3}$, from $\nu_{2}$ to $\infty$. Then the integral over $\nu_{2}$ can be performed, as in the case of the two-gluon amplitude, using an identity similar to Eq. (6.4), but with the cosines replacing the sines. The result is

$$
\begin{aligned}
I_{3}^{(1)}(\tau) & =\frac{(2 \pi)^{2}}{\tau}\left[\varepsilon_{1} \cdot \varepsilon_{2} p_{2} \cdot \varepsilon_{3}+\varepsilon_{2} \cdot \varepsilon_{3} p_{3} \cdot \varepsilon_{1}+\varepsilon_{1} \cdot \varepsilon_{3} p_{1} \cdot \varepsilon_{2}\right] \\
& \times \sum_{n=1}^{\infty}\left(\frac{1+q^{2 n}}{1-q^{2 n}}\right)^{2}+O\left(\alpha^{\prime}\right)
\end{aligned}
$$

so that the three-gluon amplitude is given by

$$
\left.A^{(1)}\left(p_{1}, p_{2}, p_{3}\right)\right|_{\mathrm{div}}=\frac{N}{4} \frac{g_{d}^{2}}{(4 \pi)^{d / 2}}\left(2 \alpha^{\prime}\right)^{2-d / 2} Z(d) A^{(0)}\left(p_{1}, p_{2}, p_{3}\right)+O\left(\alpha^{\prime}\right) \quad,
$$

and is once again expressed in terms of the integral $Z(d)$. When the integral $Z(d)$ is evaluated (as done explicitly in Appendix B), and the pinching singularities regularized, Eq. (6.11) will reduce to Eq. (4.33), as expected.

For completeness, we now show how this procedure goes through for the fourpoint amplitude. In this case we can concentrate on those terms in the amplitude that have no powers of the external momenta (and thus have a coefficient of the type $\left.\varepsilon_{i} \cdot \varepsilon_{j} \varepsilon_{h} \cdot \varepsilon_{k}\right)$. These have the correct power of $\alpha^{\prime}$ before partial integration, so we can again discard the exponentials. Then we need to compute

$$
\begin{aligned}
I_{4}^{(1)}(\tau) & =\int_{0}^{\tau} d \nu_{4} \int_{0}^{\nu_{4}} d \nu_{3} \int_{0}^{\nu_{3}} d \nu_{2}\left[\varepsilon_{1} \cdot \varepsilon_{2} \varepsilon_{3} \cdot \varepsilon_{4} \partial_{2}^{2} G\left(\nu_{2}\right) \partial_{4}^{2} G\left(\nu_{43}\right)\right. \\
& +\varepsilon_{1} \cdot \varepsilon_{3} \varepsilon_{2} \cdot \varepsilon_{4} \partial_{3}^{2} G\left(\nu_{3}\right) \partial_{4}^{2} G\left(\nu_{42}\right) \\
& \left.+\varepsilon_{1} \cdot \varepsilon_{4} \varepsilon_{3} \cdot \varepsilon_{2} \partial_{4}^{2} G\left(\nu_{4}\right) \partial_{3}^{2} G\left(\nu_{32}\right)+\ldots\right] .
\end{aligned}
$$


Using again Eq. (2.41), we can perform the integrals over the punctures, and we get

$I_{4}^{(1)}(\tau)=\frac{(2 \pi)^{2}}{\tau} \sum_{n=1}^{\infty}\left(\frac{1+q^{2 n}}{1-q^{2 n}}\right)^{2}\left[-\frac{1}{2} \varepsilon_{1} \cdot \varepsilon_{2} \varepsilon_{3} \cdot \varepsilon_{4}+\varepsilon_{1} \cdot \varepsilon_{3} \varepsilon_{2} \cdot \varepsilon_{4}-\frac{1}{2} \varepsilon_{2} \cdot \varepsilon_{3} \varepsilon_{1} \cdot \varepsilon_{4}\right]$.

The amplitude becomes then

$$
\left.A_{P}^{(1)}\left(p_{1}, p_{2}, p_{3}, p_{4}\right)\right|_{\mathrm{div}}=\frac{N}{4} \frac{g_{d}^{2}}{(4 \pi)^{d / 2}}\left(2 \alpha^{\prime}\right)^{2-d / 2} Z(d) A^{(0)}\left(p_{1}, p_{2}, p_{3}, p_{4}\right)+O\left(\alpha^{\prime}\right),
$$

where the 1PI part of the tree-level amplitude in Feynman gauge has the structure given in the square bracket of Eq. (6.13), as can easily be checked. Once again, the divergent part of the one-loop amplitude is expressed in terms of the basic integral $Z(d)$. The previous results for the tree-level and one-loop proper vertices can be obtained from the effective Lagrangian

$$
L=-\frac{1}{4} F_{\mu \nu}^{a} F_{a}^{\mu \nu}[1+K(d)],
$$

where

$$
K(d)=\frac{N}{4} \frac{g_{d}^{2}}{(4 \pi)^{d / 2}}\left(2 \alpha^{\prime}\right)^{2-d / 2} Z(d)
$$

If we now perform the limit $\alpha^{\prime} \rightarrow 0$, keeping the ultraviolet cutoff $\epsilon \equiv 2-d / 2$ small but positive, and eliminating by hand the tachyon contribution, as described in detail in Appendix B, we arrive at

$$
K(4-2 \epsilon) \rightarrow-\frac{11}{3} N \frac{g^{2}}{(4 \pi)^{2}} \frac{1}{\epsilon}+O\left(\epsilon^{0}\right) .
$$

We see that one-loop diagrams generate divergences that can be eliminated by the addition of a counterterm of the form

$$
L_{c . t .}=-\frac{1}{4} F_{\mu \nu}^{a} F_{a}^{\mu \nu} K_{A}
$$

where $K_{A}=-K$. This implies that the wave function renormalization constant is

$$
Z_{A} \equiv 1+K_{A}=1+\frac{11}{3} N \frac{g^{2}}{(4 \pi)^{2}} \frac{1}{\epsilon}
$$

in agreement with Eq. (3.13). It also implies

$$
Z_{A}=Z_{3}=Z_{4}
$$

in agreement with the results of the previous sections, and as expected in the background field method. 


\section{String in a non-abelian background field}

The results of the previous sections show that the field theory limit of string theory leads unambiguously to the background field method. Furthermore, we have learnt that contributions to the effective action can be isolated from the amplitudes by regularizing the pinching singularities in the Green function. In this section we will show how, under appropriate assumptions, these results can be derived directly, for any number of string loops, from the partition function of a string interacting with an external non-abelian background field. We will give a general expression for the planar contribution to this partition function, and we will formally generalize the analysis of Section 6 to all loops, verifying explicitly that gauge invariance is respected, and constructing a general expression for the multiloop analog of the one-loop integral $Z(d)$. We will then deal with the special problems arising at tree level and one loop, along the lines of Ref. [23], and show how the results of the previous sections are correctly reproduced with this construction. This also substantiates the somewhat formal manipulations that one has to perform on the multiloop expressions.

Let us consider the planar contribution to the partition function of an open bosonic string interacting with an external non-abelian $S U(N)$ background. It is given by

$$
\begin{aligned}
Z_{P}\left(A_{\mu}\right) & =\sum_{h=0}^{\infty} N^{h} g_{s}^{2 h-2} \int D X D g \mathrm{e}^{-S_{0}(X, g ; h)} \times \\
& \times \operatorname{Tr}\left[P_{z} \exp \left(\mathrm{i} g_{d} \int_{h} d z \partial_{z} X^{\mu}(z) A_{\mu}(X(z))\right)\right] .
\end{aligned}
$$

The path-ordering symbol $P_{z}$ reminds us that in the open string the $z$ variables are ordered, as in Eq. (2.18), along the world-sheet boundary; it is defined by

$$
\begin{aligned}
& \operatorname{Tr}\left[P_{z} \exp \left(\mathrm{i} g_{d} \int_{h} d z \partial_{z} X^{\mu}(z) A_{\mu}(X(z))\right)\right]=\sum_{n=0}^{\infty}\left(\mathrm{i} g_{d}\right)^{n} \\
\times & \int_{\Gamma_{h, n}} \prod_{i=1}^{n} d z_{i} \partial_{z_{1}} X^{\mu_{1}}\left(z_{1}\right) \ldots \partial_{z_{n}} X^{\mu_{n}}\left(z_{n}\right) \operatorname{Tr}\left[A_{\mu_{1}}\left(X\left(z_{1}\right)\right) \ldots A_{\mu_{n}}\left(X\left(z_{n}\right)\right)\right] .
\end{aligned}
$$

The precise region of integration for the punctures $z_{i}$ will in general depend on the moduli of the open Riemann surface, and we denoted it by $\Gamma_{h, n}$, for a surface of genus $h$ with $n$ punctures. The gauge coupling constant $g_{d}$ and the dimensionless string coupling $g_{s}$ are related by Eq. (2.8). Finally the bosonic string action on a genus $h$ manifold with world sheet metric $g_{\alpha \beta}$ is

$$
S_{0}(X, g ; h)=\frac{1}{4 \pi \alpha^{\prime}} \int_{h} d^{2} z \sqrt{g} g^{\alpha \beta} \partial_{\alpha} X(z) \cdot \partial_{\beta} X(z)
$$

It is convenient to separate the zero mode $x^{\mu}$ in the string coordinate $X^{\mu}$, defining

$$
X^{\mu}(z)=x^{\mu}+\left(2 \alpha^{\prime}\right)^{1 / 2} \xi^{\mu}(z)
$$


so that $\xi^{\mu}$ is dimensionless, while the zero mode $x^{\mu}$ as well as the string coordinate $X^{\mu}$ have dimensions of length. In terms of $x^{\mu}$ and $\xi^{\mu}$ the measure of the functional integral in Eq. (7.1) becomes

$$
D X=\frac{d^{d} x}{\left(2 \alpha^{\prime}\right)^{d / 2}} D \xi
$$

Inserting in Eq. (7.1) the representation of the path-ordered exponential given by Eq. (7.2), and concentrating on terms up to $O\left(2 \alpha^{\prime}\right)^{2}$, we can write the partition function as

$$
\begin{aligned}
Z_{P}\left(A_{\mu}\right) & =\sum_{h=0}^{\infty}\left(\frac{N}{(2 \pi)^{d}}\right)^{h} g_{s}^{2 h-2} \int \frac{d^{d} x}{\left(2 \alpha^{\prime}\right)^{d / 2}} \int d \mathcal{M}_{h}\{\operatorname{Tr}(1) \\
& \left.-g_{d}^{2}\left[C_{2}^{(h)}(A)+\mathrm{i} g_{d} C_{3}^{(h)}(A)+\left(\mathrm{i} g_{d}\right)^{2} C_{4}^{(h)}(A)\right]\right\},
\end{aligned}
$$

where $C_{i}^{(h)}(A)$ corresponds to the contributions of terms with $i$ external gauge fields, and is obtained performing the functional integral over the variable $\xi$.

The measure of integration over the moduli, $d \mathcal{M}_{h}$, is equal to the one given in Eq. (2.16), but does not include the differentials of the punctures, nor at this stage the factor $d V_{a b c}$, responsible for the fixing of projective invariance. For $h \geq 2$ the factor $d V_{a b c}$ can be included without problems, provided we do not fix the position of any of the punctures, choosing instead three of the fixed points of the surface in the Schottky representation. For $h=0$ this cannot be done, and for $h=1$ it can be done only partially, as the surface in these cases has less than three fixed points. One is then led to fix some of the punctures, as was done in the previous sections. In the present context, however, fixing some of the punctures would interfere with the definition of path ordering given by Eq. (7.2), and would significantly complicate the following derivation. We then choose not to fix any of the punctures, which ensures that our formulas are valid for any number of loops. The price we pay is that at tree level the expressions we write are formally infinite, because we failed to divide by the volume of the projective group. This however can be repaired by treating the projective infinities with a renormalization prescription: first we regularize them by compactifying the range of integration, then we divide by the compactified projective volume, obtaining a quantity that remains finite when the compactification radius is taken to infinity. As with any renormalization prescription, this leaves us with an undetermined finite overall constant, that can be fixed by comparing with the results of the previous sections, or with a field theory calculation. For this reason we are unable to deduce Eq. (2.8) in this functional integral approach (see also 23]). At one loop, we can partially deal with the projective invariance by fixing the positions of the two fixed points of the Schottky generator. We are then left with an overall translational invariance, that we do not eliminate by fixing one of the punctures. Rather, we integrate over all punctures, dividing at the end by the volume of translation, which is finite at one loop. This way we get the correct one-loop result, including the overall constant. 
Since Eq. (7.1) is the string expectation value of a Wilson loop winding around one boundary of the string, we expect it to be gauge invariant. It is instructive to see how gauge invariance emerges out of the calculation, for any number of string loops. To this end, we will compute separately the three contributions $C_{2}^{(h)}(A), C_{3}^{(h)}(A)$ and $C_{4}^{(h)}(A)$, and see how they correctly reconstruct the gauge invariant effective action. We will assume that pinching singularities have been regularized, and thus we will discard all terms arising from partial integrations that involve the Green function evaluated at the pinching. We will also use the periodicity of the Green function to discard all boundary terms corresponding to integrations over the complete boundary loop.

Let us start by considering $C_{2}^{(h)}(A)$. It is given by

$$
C_{2}^{(h)}(A)=\left\langle\frac{1}{2} \int d z_{1} \int d z_{2} \theta\left(z_{1}-z_{2}\right) \partial_{z_{1}} X^{\mu}\left(z_{1}\right) \partial_{z_{2}} X^{\nu}\left(z_{2}\right) A_{\mu}^{a}\left(X\left(z_{1}\right)\right) A_{\nu}^{a}\left(X\left(z_{2}\right)\right)\right\rangle
$$

where the expectation value is given by a functional integration over the field $\xi$.

Since the integrand (apart from the $\theta$-function) in Eq. (7.7) is symmetric under the exchange of the indices 1 and 2 we can also write

$$
C_{2}^{(h)}(A)=\frac{1}{4}\left\langle\int d z_{1} \int d z_{2} \partial_{z_{1}} X^{\mu}\left(z_{1}\right) \partial_{z_{2}} X^{\nu}\left(z_{2}\right) A_{\mu}^{a}\left(X\left(z_{1}\right)\right) A_{\nu}^{a}\left(X\left(z_{2}\right)\right)\right\rangle,
$$

where now $z_{1}$ and $z_{2}$ are integrated independently in the same domain.

If we now expand the gauge field around the string center of mass using Eq. (7.4), we include terms up to order $\left(2 \alpha^{\prime}\right)^{2}$, and we discard total derivatives, we get

$$
\begin{aligned}
C_{2}^{(h)}(A) & =\frac{\left(2 \alpha^{\prime}\right)^{3 / 2}}{4}\left\langle\int d z_{1} \int d z_{2} \partial_{z_{1}} \xi^{\mu}\left(z_{1}\right) \partial_{z_{2}} \xi^{\nu}\left(z_{2}\right)\right. \\
& \times\left[\xi^{\rho}\left(z_{1}\right) \partial_{\rho} A_{\mu}^{a}(x) A_{\nu}^{a}(x)+\xi^{\rho}\left(z_{2}\right) \partial_{\rho} A_{\nu}^{a}(x) A_{\mu}^{a}(x)\right. \\
& \left.\left.+\left(2 \alpha^{\prime}\right)^{1 / 2} \xi^{\rho}\left(z_{1}\right) \xi^{\sigma}\left(z_{2}\right) \partial_{\rho} A_{\mu}^{a}(x) \partial_{\sigma} A_{\nu}^{a}(x)\right]\right\rangle
\end{aligned}
$$

The expectation value can be computed simply using Wick theorem, according to

$$
<X_{\mu}(z) X_{\nu}(w)>=-g_{\mu \nu} \mathcal{G}(z, w),
$$

so that terms with an odd number of $\xi$ fields give a vanishing contribution. The result is

$$
C_{2}^{(h)}(A)=-\frac{1}{4} \tilde{F}_{\mu \nu}^{a}(x) \tilde{F}_{\mu \nu}^{a}(x)\left(2 \alpha^{\prime}\right)^{2} \int d z_{1} \int d z_{2} \theta\left(z_{1}-z_{2}\right) \partial_{z_{1}} \mathcal{G}\left(z_{1}, z_{2}\right) \partial_{z_{2}} \mathcal{G}\left(z_{1}, z_{2}\right)
$$

where $\tilde{F}_{\mu \nu}^{a}=\partial_{\mu} A_{\nu}^{a}-\partial_{\nu} A_{\mu}^{a}$ is the abelian part of the field strength tensor.

Let us now turn to terms cubic in the external field. Here we use the fact that

$$
\operatorname{Tr}\left[A_{\mu} A_{\nu} A_{\rho}\right]=\frac{1}{4}\left[\mathrm{i} f^{a b c}+d^{a b c}\right] A_{\mu}^{a} A_{\nu}^{b} A_{\rho}^{c} .
$$


The completely symmetric term does not give any contribution up to order $\left(2 \alpha^{\prime}\right)^{2}$, as one can easily see that the corresponding integrands are total derivatives. We are left with

$$
\begin{aligned}
C_{3}^{(h)}(A) & =\frac{\mathrm{i}}{4}\left(2 \alpha^{\prime}\right)^{2} f^{a b c}\left\langle\int \prod_{i=1}^{3} d z_{i} \partial_{z_{1}} \xi^{\mu}\left(z_{1}\right) \partial_{z_{2}} \xi^{\nu}\left(z_{2}\right) \partial_{z_{3}} \xi^{\rho}\left(z_{3}\right)\right. \\
& \times\left\{\xi^{\sigma}\left(z_{3}\right) A_{\mu}^{a}(x) A_{\nu}^{b}(x) \partial_{\sigma} A_{\rho}^{c}(x)\right. \\
& +\xi^{\sigma}\left(z_{2}\right) A_{\mu}^{a}(x) \partial_{\sigma} A_{\nu}^{b}(x) A_{\rho}^{c}(x) \\
& \left.\left.+\xi^{\sigma}\left(z_{1}\right) \partial_{\sigma} A_{\mu}^{a}(x) A_{\nu}^{b}(x) A_{\rho}^{c}(x)\right\}\right\rangle .
\end{aligned}
$$

Performing all possible contractions with Eq. (7.10) we arrive at

$$
\begin{aligned}
C_{3}^{(h)}(A) & =\frac{\mathrm{i}}{8}\left(2 \alpha^{\prime}\right)^{2} f^{a b c} \tilde{F}_{\mu \nu}^{a}(x) A_{\mu}^{b}(x) A_{\nu}^{c}(x) \\
& \times \int d z_{1} \int d z_{2} \int d z_{3} \theta\left(z_{1}-z_{2}\right) \theta\left(z_{2}-z_{3}\right) \\
& \times\left\{\partial_{z_{1}} \partial_{z_{2}} \mathcal{G}\left(z_{1}, z_{2}\right)\left[\partial_{z_{3}} \mathcal{G}\left(z_{2}, z_{3}\right)-\partial_{z_{3}} \mathcal{G}\left(z_{1}, z_{3}\right)\right]\right. \\
& +\partial_{z_{1}} \partial_{z_{3}} \mathcal{G}\left(z_{1}, z_{3}\right)\left[\partial_{z_{2}} \mathcal{G}\left(z_{1}, z_{2}\right)-\partial_{z_{2}} \mathcal{G}\left(z_{2}, z_{3}\right)\right] \\
& \left.+\partial_{z_{2}} \partial_{z_{3}} \mathcal{G}\left(z_{2}, z_{3}\right)\left[\partial_{z_{1}} \mathcal{G}\left(z_{1}, z_{3}\right)-\partial_{z_{1}} \mathcal{G}\left(z_{1}, z_{2}\right)\right]\right\} .
\end{aligned}
$$

This can be further simplified by partial integration of the double derivatives. Discarding all terms with Green functions evaluated at pinching, and boundary terms involving integrals around the entire boundary, we can reduce the evaluation of $C_{3}^{(h)}(A)$ to the same integral that gives $C_{2}^{(h)}(A)$,

$$
\begin{aligned}
C_{3}^{(h)}(A) & =\frac{\mathrm{i}}{2}\left(2 \alpha^{\prime}\right)^{2} f^{a b c} \tilde{F}_{\mu \nu}^{a}(x) A_{\mu}^{b}(x) A_{\nu}^{c}(x) \\
& \times \int d z_{1} \int d z_{2} \theta\left(z_{1}-z_{2}\right) \partial_{z_{1}} \mathcal{G}\left(z_{1}, z_{2}\right) \partial_{z_{2}} \mathcal{G}\left(z_{1}, z_{2}\right),
\end{aligned}
$$

which has the correct form to reconstruct the non-abelian field strength. Similar manipulations can be performed on the term quartic in the external gauge fields. In terms of Green functions, it is given by

$$
\begin{aligned}
C_{4}^{(h)}(A) & =\left(2 \alpha^{\prime}\right)^{2} \operatorname{Tr}\left(A_{\mu}(x) A_{\nu}(x) A_{\rho}(x) A_{\sigma}(x)\right) \\
& \times \int d z_{1} \int d z_{2} \int d z_{3} \int d z_{4} \theta\left(z_{1}-z_{2}\right) \theta\left(z_{2}-z_{3}\right) \theta\left(z_{3}-z_{4}\right) \\
& \times\left[g^{\mu \nu} g^{\rho \sigma} \partial_{z_{1}} \partial_{z_{2}} \mathcal{G}\left(z_{1}, z_{2}\right) \partial_{z_{3}} \partial_{z_{4}} \mathcal{G}\left(z_{3}, z_{4}\right)\right. \\
& +g^{\mu \rho} g^{\nu \sigma} \partial_{z_{1}} \partial_{z_{3}} \mathcal{G}\left(z_{1}, z_{3}\right) \partial_{z_{2}} \partial_{z_{4}} \mathcal{G}\left(z_{2}, z_{4}\right) \\
& \left.+g^{\mu \sigma} g^{\nu \rho} \partial_{z_{1}} \partial_{z_{4}} \mathcal{G}\left(z_{1}, z_{4}\right) \partial_{z_{2}} \partial_{z_{3}} \mathcal{G}\left(z_{2}, z_{3}\right)\right] .
\end{aligned}
$$


Now we observe that the group factor for the middle term in the previous equation can be written as

$$
\operatorname{Tr}\left(A_{\mu} A_{\nu} A_{\mu} A_{\nu}\right)=\operatorname{Tr}\left(A_{\mu} A_{\mu} A_{\nu} A_{\nu}\right)-\frac{1}{4} f^{b c g} A_{\mu}^{b} A_{\nu}^{c} f^{d a h} A_{\mu}^{d} A_{\nu}^{a}
$$

It is easy to see that only the last term in Eq. (7.17) contributes to Eq. (7.16): in fact, in the sum of the other three terms of Eq. (7.16) the integrand is completely symmetric in the exchange of any two punctures. The theta functions can thus be removed, and then the integrand is a total derivative. The only surviving contribution is

$$
\begin{aligned}
C_{4}^{(h)}(A) & =-\frac{1}{4}\left(2 \alpha^{\prime}\right)^{2} f^{b c g} A_{\mu}^{b}(x) A_{\nu}^{c}(x) f^{d a g} A_{\mu}^{d}(x) A_{\nu}^{a}(x) \\
& \times \int d z_{1} \int d z_{2} \int d z_{3} \int d z_{4} \theta\left(z_{1}-z_{2}\right) \theta\left(z_{2}-z_{3}\right) \theta\left(z_{3}-z_{4}\right) \\
& \times \partial_{z_{1}} \partial_{z_{3}} \mathcal{G}\left(z_{1}, z_{3}\right) \partial_{z_{2}} \partial_{z_{4}} \mathcal{G}\left(z_{2}, z_{4}\right) .
\end{aligned}
$$

Partial integration gives finally

$$
\begin{aligned}
C_{4}^{(h)}(A) & =\frac{1}{4}\left(2 \alpha^{\prime}\right)^{2} f^{b c g} A_{\mu}^{b}(x) A_{\nu}^{c}(x) f^{d a g} A_{\mu}^{d}(x) A_{\nu}^{a}(x) \\
& \times \int d z_{2} \int d z_{3} \theta\left(z_{2}-z_{3}\right) \partial_{z_{2}} \mathcal{G}\left(z_{2}, z_{3}\right) \partial_{z_{3}} \mathcal{G}\left(z_{2}, z_{3}\right)
\end{aligned}
$$

As expected, $C_{2}^{(h)}(A), C_{3}^{(h)}(A)$ and $C_{4}^{(h)}(A)$ combine to reconstruct the non-abelian field strength. Our results can be summarized in a very compact expression for the partition function defined by Eq. (7.1), valid for any number of string loops. Omitting the vacuum contribution and higher orders in $\alpha^{\prime}$, we get

$$
Z_{P}\left(A_{\mu}\right)=\left(2 \alpha^{\prime}\right)^{2-d / 2} \int d^{d} x\left[-\frac{1}{4} F_{\mu \nu}^{a}(x) F_{\mu \nu}^{a}(x)\right] \sum_{h=0}^{\infty} N^{h} g_{s}^{2 h-2} Z^{(h)}(d)
$$

where

$$
Z^{(h)}(d)=-g_{d}^{2} \int d \mathcal{M}_{h} \int d z_{1} \int d z_{2} \theta\left(z_{1}-z_{2}\right) \partial_{z_{1}} \mathcal{G}\left(z_{1}, z_{2}\right) \partial_{z_{2}} \mathcal{G}\left(z_{1}, z_{2}\right)
$$

and

$$
F_{\mu \nu}^{a}=\partial_{\mu} A_{\nu}^{a}-\partial_{\nu} A_{\mu}^{a}+g_{d} f^{a b c} A_{\mu}^{b} A_{\nu}^{c}
$$

Up to some constant prefactors, $Z^{(h)}(d)$ is the multiloop generalization of the integral $Z(d)$ defined in Section 6 .

As we mentioned before, tree-level and one-loop contributions need extra care because of the unfixed projective invariance of the functional integral. Here we sketch how this problem can be handled, drawing on the results of the previous sections and on the ideas of [23. In the process, as expected, we rederive the one-loop integral $Z(d)$. 
At tree level, the projective infinity that would have been eliminated by fixing three of the punctures can be regularized by compactifying the integration region of the variables $z_{i}$, mapping them from the real axis to a circle. On a circle, following Ref. [23], we can use the Green function

$$
\begin{aligned}
\hat{G}\left(\phi_{1}, \phi_{2}\right) & =\log \left[2 \mathrm{i} \sin \left(\frac{\phi_{1}-\phi_{2}}{2}\right)\right] \\
& =-\sum_{n=1}^{\infty} \frac{\cos n\left(\phi_{1}-\phi_{2}\right)}{n}+\ldots .
\end{aligned}
$$

The integrals over the punctures $\phi_{i}$ are now ordered in the interval $(0,2 \pi)$. The dots in Eq. (7.23) stand for terms independent of the punctures, that are irrelevant.

Using Eq. (7.23), we find that the basic integral appearing in $Z^{(0)}(d)$ is given by

$$
\begin{aligned}
& \int_{0}^{2 \pi} d \phi_{1} \int_{0}^{\phi_{1}} d \phi_{2} \partial_{\phi_{1}} \hat{G}\left(\phi_{1}, \phi_{2}\right) \partial_{\phi_{2}} \hat{G}\left(\phi_{1}, \phi_{2}\right) \\
= & -\frac{2 \pi}{2} \int_{0}^{2 \pi} d \phi \sum_{n=1}^{\infty} \sin ^{2} n \phi=-\frac{(2 \pi)^{2}}{4} \sum_{n=1}^{\infty} 1=\frac{(2 \pi)^{2}}{8},
\end{aligned}
$$

where we have regularized the divergent sum using Eq. (5.29).

Inserting this result in Eq. (7.11) we get, at tree level,

$$
C_{2}^{(0)}(A)=-\frac{1}{4} \tilde{F}_{\mu \nu}^{a}(x) \tilde{F}_{\mu \nu}^{a}(x)\left(2 \alpha^{\prime}\right)^{2} \frac{(2 \pi)^{2}}{8}
$$

If we compute the six integrals in Eq. (7.14), and the three integrals in Eq. (7.16), we see that the combination of $C_{2}^{(0)}(A), C_{3}^{(0)}(A)$ and $C_{4}^{(0)}(A)$ in Eq. (7.6) correctly reconstructs the full non-abelian gauge invariant action, in agreement with the results of Ref. [23], where only $C_{2}^{(0)}(A)$ is explicitly computed while $C_{3}^{(0)}(A)$ and $C_{4}^{(0)}(A)$ are deduced from gauge invariance. The result is of the form

$$
Z_{P}^{h=0}\left(A_{\mu}\right)=-\frac{v}{4} \int d^{d} x\left[F_{\mu \nu}^{a}(x) F_{\mu \nu}^{a}(x)\right]
$$

where, with this regularization, $v=-2 \pi^{2}$ in four dimensions. Notice that $v$ turns out to equal the integration volume multiplied with $\zeta(0)$, and is clearly regularization-dependent.

The one-loop coefficients $C_{i}^{(1)}(A)$ can be similarly computed without fixing any of the punctures. We can however fix the location of the fixed points $\eta$ and $\xi$ of the Schottky group, as was done in the previous sections. Having done that, we are left with invariance under translations of the $z_{i}$, or, more conveniently, of the variables $\nu_{i}$, related to the $z_{i}$ by Eq. (2.30). The difference with respect to the tree level integrals is that now the variables $\nu_{i}$ are integrated in an ordered way in the interval $(0, \tau)$ instead of $(0,2 \pi)$. As a consequence, instead of the factor $2 \pi$ arising from the redundant integration of a translationally invariant function, we will get a 
factor $\tau$. We have to remember, however, to divide the computed expression by the volume of the translational part of the projective group, that has not been fixed, and that is equal to $\int_{k}^{1} \frac{d z}{z}=-\log k=2 \tau$. With the inclusion of this factor the measure of integration over $k$ is

$$
d \mathcal{M}_{1}=\frac{d k}{k^{2}}\left[-\frac{1}{2 \pi} \log k\right]^{-d / 2} \frac{1}{(-\log k)} \prod_{n=1}^{\infty}\left(1-k^{n}\right)^{2-d} .
$$

The coefficients $C_{2}^{(1)}(A), C_{3}^{(1)}(A)$ and $C_{4}^{(1)}(A)$ can be computed directly from Eqs. (7.11), (7.14) and (7.16), so that no extra assumptions on the cancellation of boundary terms need to be made. We find, using the Green function given by Eq. (2.41),

$$
C_{i}^{(1)}(A)=-2 C_{i}^{(0)}(A) \sum_{n=1}^{\infty}\left(\frac{1+q^{2 n}}{1-q^{2 n}}\right)^{2},
$$

for $i=2,3,4$. The one-loop contribution to the partition function defined by Eq. (7.1) is thus given by

$$
Z_{P}^{h=1}\left(A_{\mu}\right)=\frac{N}{4} \frac{g_{d}^{2}}{(4 \pi)^{d / 2}}\left(2 \alpha^{\prime}\right)^{2-d / 2} Z(d) \int d^{d} x\left[-\frac{1}{4} F_{\mu \nu}^{a}(x) F_{\mu \nu}^{a}(x)\right],
$$

which is precisely the result of Eqs. (6.15) and (6.16), with $Z(d)$ given in Eq. (6.7). We have thus verified that the general formalism developed in the first part of this section applies to the somewhat special cases $h=0$ and $h=1$, and we have also verified that the assumptions made for general $h$, concerning the cancellation of boundary terms and the regularization of the pinchings, are satisfied for $h<2$. Notice finally that the present functional integral derivation of the renormalization constants provides an alternative way for computing the normalization factor $C_{h}$ given in Eq. (2.4). The $h$-loop contribution to $Z_{P}(A)$ contains in fact precisely a factor of $(2 \pi)^{(-d h)}$ corresponding to the integration over $h$ loop momenta, a factor of $\left(2 \alpha^{\prime}\right)^{-d / 2}$ from the change of measure given by Eq. (7.5), and the correct power of the string coupling constant.

\section{Summary and perspectives}

We have analyzed in detail three related methods to calculate renormalization constants in Yang-Mills theory, all based on a consistent prescription to continue off the mass shell multigluon amplitudes derived from string theory. Our prescription leads unambiguously to the background field method, and is phrased directly in the language of dimensional regularization.

The use of the open bosonic string has introduced undesired difficulties due to the presence of a tachyon, but we have shown explicitly how such difficulties can be overcome, at least at one loop. In this context, perhaps the most significant observation is that divergences associated with the tachyon are not regularized by 
analytic continuation of the space-time dimension, whereas all divergences associated with Yang-Mills gauge bosons turn into the usual poles at $d=4$. On the other hand, using the simplest available string theory has allowed us to phrase our technique directly in the language of multiloop string amplitudes, while minimizing the amount of string technology needed for the calculations. This may prove necessary when practical multiloop calculations are attempted.

Our work complements what is known about string-derived on-shell multigluon amplitudes [8], and effective actions [2], in several ways. Our prescription, clearly tied to, and in fact inspired by the background field method in field theory, provides an off-shell extension of the analysis of Ref. [11]. By working with a simple string theory, and by not performing the partial integration of double derivatives of the world-sheet Green functions that appear in the amplitudes, we have been able to map explicitly each region of moduli space that contributes to the field theory limit to a specific set of Feynman diagrams. This mapping, which may prove useful in future applications going beyond one loop, has enabled us to identify precisely the regions of moduli space that contribute to the effective action, leading to considerable simplifications in the calculation of renormalization constants. At one loop, ultraviolet divergences of gluon amplitudes turn out to be summarized by a single integral over the string modular parameter, which still contains all contributions from the infinite tower of massive string states. The field theory limit of this integral is the gluon wave function renormalization of the background field method. We have thus made a precise connection between the techniques of Ref. [8] and those of Ref. [2]. In particular, we have shown how the $\zeta$-function regularization of pinching singularities introduced in Ref. [16] is precisely suited to handle correctly the contact terms left over by the propagation of tachyons in pinched configurations. Finally, we have studied the partition function of an open string interacting with a background Yang-Mills field, to all orders in string perturbation theory, focusing on planar diagrams. As might have been expected from the explicit results obtained at tree level and at one loop, this partition function is a generating functional for the contributions to the gluon effective action of string diagrams with an arbitrary number of legs and loops. A formal analysis of this partition function shows how string theory generates a gauge-invariant effective action for any number of loops, and in fact leads to a multiloop generalization of the one-loop integral, $Z(d)$, generating the renormalization constants. Tree-level and one-loop diagrams are special, because of the extra invariances that reduce the dimensionality of the corresponding string moduli space, but the differences can be handled and the known results are recovered also from the general $h$-loop formalism.

The general formalism developed in Section 7 helps perhaps to shed some light on the relationship between string theory and the background field method. Examining the expression for the string partition function, Eq. (7.1), one may observe that any string amplitude may be written in terms of interactions with a background field, provided the bakground field satisfies the string equations of motion. In particular, ordinary gluon amplitudes correspond to a background field constructed out of plane 
waves. The only apparent obstacle to this interpretation is the presence in the full string amplitudes of the pinching singularities, that correspond to interactions of the external fields happening far away (on the string scale) from the string loops. This is exactly the same problem that arises in field theory, in background field calculations of $S$-matrix elements. There, if one allows for interactions among the background fields, one is forced to quantize them also, introducing a new gauge fixing term and constructing the corresponding propagator. Here, once the pinching singularities are removed, the calculation of the effective action performed along the lines of Section 6, or of Section 0, can be simply understood as an infrared limit of the calculation of a gluon scattering amplitude, where a quasi-constant background field has taken the place of the plane wave representing the gluon.

A few remarks may be added concerning the choice of regularization scheme. This paper makes extensive use of dimensional regularization, which is very practical if one whishes to compare the results with QCD calculation, where it is by far the most commonly used scheme. However, it should be kept in mind that this choice is not by any means the only possible one. In fact, it may not be the best choice if one wishes to extend to more than one loop the effective action calculations performed in Section 6. In general, $h$-loop contributions to renormalization constants appear in dimensional regularization as coefficients of the single pole in $\epsilon=2-d / 2$, and may be missed if one uses a method that neglects $O(\epsilon)$ corrections. Regularization with, say, a momentum space cutoff may be more appropriate, since in that case divergences are further classified according to the power of the cutoff involved (see for example Ref. [2]). It may also be argued that string theory by itself provides a regularized version of Yang-Mills theory, with the role of the cutoff being played by $1 / \alpha^{\prime}$. In fact, this "stringy" regularization is quite similar in spirit to Pauli-Villars regularization, in the sense that the theory is made finite by adding an infinite number of new degrees of freedom with heavy masses and with carefully selected coefficients. It might be instructive to study how this "stringy" regularization translates in a field theory language.

Summarizing, we believe we have shown that the multiloop string technology needed to study perturbative Yang-Mills theory exists, and the complexity of the necessary calculations may remain manageable, provided one uses a sufficiently simple string theory, as we have done in this paper. This may have wide-ranging applications, not only to practical calculations of phenomenological interest, but more generally to develop a deeper understanding of the organization of the perturbative expansion of gauge theories. String theory has proven to be remarkably clever in organizing gauge theory amplitudes both at tree level and at one loop, suggesting the best choices of color and helicity organizations, as well as the best combination of gauges. It is fair to hope that similar results may be achieved at least at two loops. On the other hand, the very existence of explicit all-order expressions for the amplitudes in string theory suggests that these tools might also be useful to derive all-order results in field theory, as was mentioned in Section 3. A simple example of such a derivation is given by our discussion of string propagation 
in a background field.

\section{Acknowledgements}

One of us (R. M.) would like to thank NORDITA for the kind hospitality during the completion of this work. L. M. thanks G. Marchesini for a stimulating remark and D. Dunbar for instructive conversations. This research was partially supported by the EU, within the framework of the program "Gauge Theories, Applied Supersymmetry and Quantum Gravity", under contract SCI-CT92-0789.

\section{Appendix A}

In this appendix we compute the normalization coefficients of the $h$-loop string amplitudes, $C_{h}$, and of the gluon states, $\mathcal{N}_{0}$, given respectively in Eqs. (2.4) and (2.7). Our strategy to do so is the following: we first compute the three and fourgluon amplitudes at tree level, and require that in the limit $\alpha^{\prime} \rightarrow 0$ they reduce to the corresponding amplitudes derived from the Yang-Mills Lagrangian. This fixes $C_{0}$ and $\mathcal{N}_{0}$ as functions of the Yang-Mills coupling constant $g_{d}$; we then check that these normalizations are consistent with the factorization properties required by unitarity, and finally we implement the sewing procedure to obtain the multiloop amplitudes, and their overall normalization $C_{h}$.

As discussed in section 4 , in the open bosonic string the color-ordered threegluon amplitude at tree level is

$$
\begin{aligned}
A^{(0)}\left(p_{1}, p_{2}, p_{3}\right)= & C_{0} \mathcal{N}_{0}^{3} \operatorname{Tr}\left(\lambda^{a_{1}} \lambda^{a_{2}} \lambda^{a_{3}}\right) \sqrt{2 \alpha^{\prime}}\left(-\varepsilon_{1} \cdot \varepsilon_{2} p_{2} \cdot \varepsilon_{3}\right. \\
& \left.-\varepsilon_{2} \cdot \varepsilon_{3} p_{3} \cdot \varepsilon_{1}-\varepsilon_{3} \cdot \varepsilon_{1} p_{1} \cdot \varepsilon_{2}+O\left(\alpha^{\prime}\right)\right),
\end{aligned}
$$

where $\varepsilon_{i}, p_{i}, a_{i}$ are the polarization, the momentum and the color index of the $i$-th gluon, and the $\lambda$ 's are the generators of $S U(N)$ in the fundamental representation. For them we take the standard field theory conventions, i.e.

$$
\operatorname{Tr}\left(\lambda^{a} \lambda^{b}\right)=\frac{1}{2} \delta^{a b} \quad, \quad \operatorname{Tr}\left(\lambda^{a}\left[\lambda^{b}, \lambda^{c}\right]\right)=\frac{\mathrm{i}}{2} f^{a b c}
$$

where $f^{a b c}$ are the group structure constants. We notice that this choice implies in particular that

$$
\sum_{a} \operatorname{Tr}\left(A \lambda^{a}\right) \operatorname{Tr}\left(\lambda^{a} B\right)=\frac{1}{2} \operatorname{Tr}(A B)-\frac{1}{2 N} \operatorname{Tr}(A) \operatorname{Tr}(B)
$$




$$
\sum_{a} \operatorname{Tr}\left(\lambda^{a} A \lambda^{a}\right)=\frac{N}{2} \operatorname{Tr}(A)-\frac{1}{2 N} \operatorname{Tr}(A)
$$

with $A$ and $B$ being arbitrary $N \times N$ matrices.

With this normalization of the color matrices, it is easy to check that in the limit $\alpha^{\prime} \rightarrow 0$, Eq. (A.1) reduces to the color-ordered three-gluon amplitude derived from the Yang-Mills Lagrangian if

$$
C_{0} \mathcal{N}_{0}^{3}=\frac{4 g_{d}}{\sqrt{2 \alpha^{\prime}}}
$$

where $g_{d}$ is the (dimensionful) Yang-Mills coupling constant in $d$ dimensions.

The color-ordered four-gluon amplitude at tree level is

$$
\begin{aligned}
A^{(0)}\left(p_{1}, p_{2}, p_{3}, p_{4}\right)= & C_{0} \mathcal{N}_{0}^{4} \operatorname{Tr}\left(\lambda^{a_{1}} \lambda^{a_{2}} \lambda^{a_{3}} \lambda^{a_{4}}\right) \frac{\Gamma\left(1-\alpha^{\prime} s\right) \Gamma\left(1-\alpha^{\prime} t\right)}{\Gamma\left(1+\alpha^{\prime} u\right)} \\
& {\left[\frac{u}{s\left(1+\alpha^{\prime} s\right)} \varepsilon_{1} \cdot \varepsilon_{2} \varepsilon_{3} \cdot \varepsilon_{4}+\frac{1}{1+\alpha^{\prime} u} \varepsilon_{1} \cdot \varepsilon_{3} \varepsilon_{2} \cdot \varepsilon_{4}\right.} \\
& +\frac{u}{t\left(1+\alpha^{\prime} t\right)} \varepsilon_{1} \cdot \varepsilon_{4} \varepsilon_{2} \cdot \varepsilon_{3} \\
& -\frac{2}{t}\left(\varepsilon_{1} \cdot \varepsilon_{3} \varepsilon_{4} \cdot p_{1} \varepsilon_{2} \cdot p_{3}+\varepsilon_{1} \cdot \varepsilon_{4} \varepsilon_{3} \cdot p_{1} \varepsilon_{2} \cdot p_{4}\right. \\
& \left.\quad+\varepsilon_{2} \cdot \varepsilon_{3} \varepsilon_{1} \cdot p_{3} \varepsilon_{4} \cdot p_{2}+\varepsilon_{2} \cdot \varepsilon_{4} \varepsilon_{3} \cdot p_{2} \varepsilon_{1} \cdot p_{4}\right) \\
& -\frac{2}{s}\left(\varepsilon_{1} \cdot \varepsilon_{2} \varepsilon_{4} \cdot p_{2} \varepsilon_{3} \cdot p_{1}+\varepsilon_{1} \cdot \varepsilon_{3} \varepsilon_{4} \cdot p_{3} \varepsilon_{2} \cdot p_{1}\right. \\
& \left.\quad+\varepsilon_{2} \cdot \varepsilon_{4} \varepsilon_{3} \cdot p_{4} \varepsilon_{1} \cdot p_{2}+\varepsilon_{3} \cdot \varepsilon_{4} \varepsilon_{1} \cdot p_{3} \varepsilon_{2} \cdot p_{4}\right) \\
& -\frac{2 u}{s t}\left(\varepsilon_{1} \cdot \varepsilon_{2} \varepsilon_{4} \cdot p_{1} \varepsilon_{3} \cdot p_{2}+\varepsilon_{1} \cdot \varepsilon_{4} \varepsilon_{3} \cdot p_{4} \varepsilon_{2} \cdot p_{1}\right. \\
& \left.\quad+\varepsilon_{2} \cdot \varepsilon_{3} \varepsilon_{1} \cdot p_{2} \varepsilon_{4} \cdot p_{3}+\varepsilon_{3} \cdot \varepsilon_{4} \varepsilon_{2} \cdot p_{3} \varepsilon_{1} \cdot p_{4}\right) \\
& \left.+O\left(\alpha^{\prime}\right)\right] \quad
\end{aligned}
$$

where, as usual, $s=-\left(p_{1}+p_{2}\right)^{2}, t=-\left(p_{1}+p_{4}\right)^{2}$ and $u=-\left(p_{1}+p_{3}\right)^{2}$. In the limit $\alpha^{\prime} \rightarrow 0$ this expression reduces to the color-ordered four-gluon amplitude (consisting of both 1PI and exchange diagrams in the $s$ and $t$ channels) derived from the Yang-Mills lagrangian if

$$
C_{0} \mathcal{N}_{0}^{4}=4 g_{d}^{2} .
$$

Combining Eq. (A.5) and Eq. (A.7), we easily derive

$$
\begin{aligned}
& \mathcal{N}_{0}=g_{d} \sqrt{2 \alpha^{\prime}}, \\
& C_{0}=\frac{1}{\left(g_{d} \alpha^{\prime}\right)^{2}} .
\end{aligned}
$$


We now verify that these normalizations are consistent with the factorization properties required by unitarity. To do so, let us consider the amplitude given in Eq. (A.6), which has poles at

$$
s=m_{I}^{2} \equiv \frac{I}{\alpha^{\prime}} \quad \text { or } \quad t=m_{I}^{2}
$$

for $I=-1,0,1, \ldots$. Each pole is associated with the exchange of an intermediate string state $|I\rangle$ of mass $m_{I}$ in the $s$ or in the $t$ channel $(I=-1$ corresponding to tachyon exchange, $I=0$ to gluon exchange, and so forth). Unitarity requires that the residues of these poles have certain simple factorization properties. As far as the $s$ channel is concerned, these can be symbolically represented by saying that, for $s \rightarrow m_{I}^{2}$,

$$
A^{(0)}\left(p_{1}, p_{2}, p_{3}, p_{4}\right) \sim \sum_{(I)} A_{I}^{(0)}\left(p_{1}, p_{2}, q\right) \frac{1}{q^{2}+m_{I}^{2}} A_{I}^{(0)}\left(-q, p_{3}, p_{4}\right)
$$

In this formula $q=-p_{1}-p_{2}=p_{3}+p_{4}$ is the exchanged momentum, while $A_{I}^{(0)}\left(p_{1}, p_{2}, q\right)$ denotes the ordered amplitude involving two gluons with momenta $p_{1}$ and $p_{2}$, and the $I$-th string state with momentum $q$. The symbol $\sum_{(I)}$ stands for a sum over all these intermediate states, including for each one of them a sum over all its quantum numbers.

Eq. (A.11) is an example of the well-known fact that a four-string amplitude can be interpreted as a sewing of two three string amplitudes, with $1 /\left(q^{2}+m_{I}^{2}\right)$ acting as a propagator for the $I$-th state $|I\rangle$. Notice that

$$
\frac{1}{q^{2}+m_{I}^{2}}|I\rangle=\frac{\alpha^{\prime}}{L_{0}-1}|I\rangle,
$$

where $L_{0}$ is the zero mode of the Virasoro algebra. This equation allows to identify $\alpha^{\prime} /\left(L_{0}-1\right)$ as the sewing operator ${ }^{6}$. Sice we have been concentrating on planar contributions, in the sewing process we must be careful to preserve the ordering of the color indices, i.e. the sewing must also occur in a planar way. For $S U(N)$ this is realized by retaining in the combinations of the group color factors only those terms which are leading in the formal limit $N \rightarrow \infty$.

To check whether Eq. (A.11) is satisfied or not, we first examine the gluon exchange $(I=0)$. From Eq. (A.6) we easily see that when $s \rightarrow 0$

$$
\begin{aligned}
A^{(0)}\left(p_{1}, p_{2}, p_{3}, p_{4}\right) & \sim 2 C_{0} \mathcal{N}_{0}^{4} \operatorname{Tr}\left(\lambda^{a_{1}} \lambda^{a_{2}} \lambda^{a_{3}} \lambda^{a_{4}}\right) \frac{1}{s} \\
& \times\left[-\frac{t}{2} \varepsilon_{1} \cdot \varepsilon_{2} \varepsilon_{3} \cdot \varepsilon_{4}-\varepsilon_{1} \cdot \varepsilon_{2} \varepsilon_{4} \cdot p_{2} \varepsilon_{3} \cdot p_{1}-\varepsilon_{1} \cdot \varepsilon_{3} \varepsilon_{4} \cdot p_{3} \varepsilon_{2} \cdot p_{1}\right.
\end{aligned}
$$

\footnotetext{
${ }^{6}$ We refer to [18] for a thorough discussion of the sewing procedure and for an analysis of different forms of sewing operators, including their expressions when the ghost degrees of freedom are taken into account.
} 


$$
\begin{aligned}
& -\varepsilon_{2} \cdot \varepsilon_{4} \varepsilon_{3} \cdot p_{4} \varepsilon_{1} \cdot p_{2}-\varepsilon_{3} \cdot \varepsilon_{4} \varepsilon_{1} \cdot p_{3} \varepsilon_{2} \cdot p_{4}+\varepsilon_{1} \cdot \varepsilon_{2} \varepsilon_{4} \cdot p_{1} \varepsilon_{3} \cdot p_{2} \\
& +\varepsilon_{1} \cdot \varepsilon_{4} \varepsilon_{3} \cdot p_{4} \varepsilon_{2} \cdot p_{1}+\varepsilon_{2} \cdot \varepsilon_{3} \varepsilon_{1} \cdot p_{2} \varepsilon_{4} \cdot p_{3}+\varepsilon_{3} \cdot \varepsilon_{4} \varepsilon_{2} \cdot p_{3} \varepsilon_{1} \cdot p_{4} \\
& \left.+O\left(\alpha^{\prime}\right)\right] .
\end{aligned}
$$

On the other hand, using Eq. (A.1) for $A_{0}^{(0)}$ and writing explicitly the sum over the intermediate gluons as a sum over colors and polarizations, we have

$$
\begin{aligned}
& \sum_{(0)} A_{0}^{(0)}\left(p_{1}, p_{2}, q\right) \frac{1}{q^{2}} A_{0}^{(0)}\left(-q, p_{3}, p_{4}\right) \\
= & \frac{C_{0}^{2} \mathcal{N}_{0}^{6} 2 \alpha^{\prime}}{q^{2}} \sum_{b}\left(\operatorname{Tr}\left(\lambda^{a_{1}} \lambda^{a_{2}} \lambda^{b}\right) \operatorname{Tr}\left(\lambda^{b} \lambda^{a_{3}} \lambda^{a_{4}}\right)\right) \\
\times & \sum_{\varepsilon}\left[\left(\varepsilon_{1} \cdot \varepsilon_{2} p_{2} \cdot \varepsilon+\varepsilon_{2} \cdot \varepsilon q \cdot \varepsilon_{1}+\varepsilon \cdot \varepsilon_{1} p_{1} \cdot \varepsilon_{2}+O\left(\alpha^{\prime}\right)\right)\right. \\
& \left.\left(\varepsilon \cdot \varepsilon_{3} p_{3} \cdot \varepsilon_{4}+\varepsilon_{3} \cdot \varepsilon_{4} p_{4} \cdot \varepsilon-\varepsilon_{4} \cdot \varepsilon q \cdot \varepsilon_{3}+O\left(\alpha^{\prime}\right)\right)\right] .
\end{aligned}
$$

We now use the identity

$$
\sum_{\varepsilon} a \cdot \varepsilon b \cdot \varepsilon=a \cdot b
$$

and perform the sum over colors using Eq. (A.3), and keeping only the planar contribution. After some straightforward algebra, it is easy to see that Eq. (A.13) and Eq. (A.14) coincide if

$$
C_{0} \mathcal{N}_{0}^{2} \alpha^{\prime}=2,
$$

which is satisfied by $\mathcal{N}_{0}$ and $C_{0}$, as given in Eqs. (A.8) and (A.9).

It is instructive to examine also the contributions due to exchanges of other string states. For example, let us consider the tachyon $(I=-1)$. In order to check Eq. (A.11) in this case, we need to know the ordered amplitude $A_{-1}^{(0)}$ involving two gluons and one tachyon. This can be easily computed in the operator formalism, and one finds

$$
A_{-1}^{(0)}\left(p_{1}, p_{2}, q\right)=C_{0} \mathcal{N}_{0}^{2} \mathcal{N}_{-1} \operatorname{Tr}\left(\lambda^{a_{1}} \lambda^{a_{2}} \lambda^{b}\right)\left(\varepsilon_{1} \cdot \varepsilon_{2}+O\left(\alpha^{\prime}\right)\right)
$$

where $\mathcal{N}_{-1}$ denotes the normalization of the tachyon state. Repeating the same steps as before, we can see that the residue of the four-gluon amplitude at the tachyon pole, Eq. (A.6), factorizes into the product of two three-point amplitudes, like Eq. (A.17), if $C_{0} \mathcal{N}_{-1}{ }^{2} \alpha^{\prime}=2$. Without much difficulty it is possible to generalize this relation to the $I$-th string state, with normalization $\mathcal{N}_{I}$, and thus one can conclude that unitarity implies that

$$
C_{0} \mathcal{N}_{I}^{2} \alpha^{\prime}=2
$$

for all $I$. 
Let us now turn to the normalization $C_{1}$ of the one-loop amplitudes. Since a loop can be obtained by sewing two legs of a tree-level amplitude, the coefficient $C_{1}$ can be deduced from $C_{0}$. To see this, let us consider a color-ordered amplitude for the scattering of $M$ gluons and two arbitrary states $I$ and $J$ (which for simplicity we take to be adjacent in the chosen ordering along the loop), at tree level. We schematically denote such amplitude by

$$
A_{I J}^{(0)}\left(p_{I}, p_{J}\right)=C_{0} \mathcal{N}_{I} \mathcal{N}_{0}{ }^{M} \mathcal{N}_{J} \operatorname{Tr}\left(\lambda^{b_{I}} \lambda^{a_{1}} \cdots \lambda^{a_{M}} \lambda^{b_{J}}\right) K_{I J}\left(p_{I}, p_{J}\right),
$$

where $K_{I J}\left(p_{I}, p_{J}\right)$ is an appropriate kinematic factor. To obtain the $M$-gluon amplitude at one loop we first identify the states $I$ and $J$ by means of the sewing operator, which carries a factor of $\alpha^{\prime}$, and then we integrate over their momentum $q$ and sum over their color index $b$. Finally, we must sum over all possible intermediate states. Thus, upon sewing, Eq. (A.19) is replaced by

$$
A^{(1)}\left(p_{1}, \ldots p_{M}\right)=\sum_{J} C_{0} \mathcal{N}_{J}^{2} \mathcal{N}_{0}^{M} \sum_{b} \operatorname{Tr}\left(\lambda^{b} \lambda^{a_{1}} \ldots \lambda^{a_{M}} \lambda^{b}\right) \int \frac{d^{d} q}{(2 \pi)^{d}} \tilde{K}_{J J}(q, q)
$$

where $\tilde{K}_{J J}(q, q)$ is what it is obtained from $K_{J J}(q, q)$ with the inclusion of the sewing operator. The integral over $q$ produces a factor of $\left(2 \alpha^{\prime}\right)^{-d / 2}$ and leaves the non-zero mode part of $\tilde{K}_{J J}(q, q)$, which we denote by $\tilde{K}_{J J}^{\prime}$, whereas the sum over colors yields

$$
\sum_{b} \operatorname{Tr}\left(\lambda^{b} \lambda^{a_{1}} \cdots \lambda^{a_{M}} \lambda^{b}\right)=\frac{N}{2} \operatorname{Tr}\left(\lambda^{a_{1}} \cdots \lambda^{a_{M}}\right),
$$

provided we use Eq. (A.4), and keep only the planar contribution. Then, using Eq. (A.18), Eq. (A.20) becomes

$$
A_{P}^{(1)}\left(p_{1}, \ldots p_{M}\right)=\frac{1}{(2 \pi)^{d}}\left(2 \alpha^{\prime}\right)^{-d / 2} \mathcal{N}_{0}^{M} N \operatorname{Tr}\left(\lambda^{a_{1}} \cdots \lambda^{a_{M}}\right) \sum_{J} \tilde{K}_{J J}^{\prime} .
$$

This is the schematic expression of the $M$-gluon planar amplitude at one loop, and from it we can deduce that

$$
C_{1}=\frac{1}{(2 \pi)^{d}}\left(2 \alpha^{\prime}\right)^{-d / 2}
$$

When we iterate this sewing procedure to generate more loops, we realize that

$$
\frac{C_{h}}{C_{h-1}}=\frac{1}{(2 \pi)^{d}}\left(2 \alpha^{\prime}\right)^{-d / 2} g_{d}^{2} \alpha^{\prime 2}
$$

which leads to

$$
C_{h}=\frac{1}{(2 \pi)^{d h}}\left(2 \alpha^{\prime}\right)^{-d h / 2} g_{d}^{2 h-2}\left(\alpha^{\prime}\right)^{2 h-2} .
$$

If we introduce the dimensionless string coupling constant

$$
g_{s}=\frac{g_{d}}{2}\left(2 \alpha^{\prime}\right)^{1-d / 4},
$$


it is possible to rewrite Eq. (A.25) as

$$
C_{h}=\frac{1}{(2 \pi)^{d h}} g_{s}^{2 h-2} \frac{1}{\left(2 \alpha^{\prime}\right)^{d / 2}}
$$

All factors in this expression have a simple interpretation: the first ensures the canonical normalization of all loop momentum integrals, the second contains the correct power of the string coupling costant, dictated by string perturbation theory, and finally the last is necessary to give the vacuum amplitude the proper dimension, $(\text { length })^{-d}$.

\section{Appendix B}

In this appendix we compute the integral $Z(d)$, Eq. (6.7), in the field theory limit, and thus derive Eq. (6.17) from Eq. (6.16).

The first important step is the observation that the sum appearing in $Z(d)$ can be written as

$$
\sum_{n=1}^{\infty}\left(\frac{1+q^{2 n}}{1-q^{2 n}}\right)^{2}=\sum_{n=1}^{\infty}\left[1+\frac{4 q^{2 n}}{\left(1-q^{2 n}\right)^{2}}\right]=-2 q \frac{d}{d q} \log \left[q^{1 / 4} \prod_{n=1}^{\infty}\left(1-q^{2 n}\right)\right]
$$

where, following Ref. [2], we have regularized the divergent sum $\sum_{n=1}^{\infty} 1$ by means of Eq. (5.29).

It is interesting then to notice that the result of the integration over the punctures in the open string becomes very similar to what has been found by Kaplunovski [5] for the closed heterotic string.

Using the identity

$$
f\left(q^{2}\right) \equiv \prod_{n=1}^{\infty}\left(1-q^{2 n}\right)=\left(-\frac{2 \pi}{\log k}\right)^{-1 / 2} k^{1 / 24} \mathrm{e}^{-\pi^{2} /(6 \log k)} f(k)
$$

with $k \equiv \mathrm{e}^{-2 \tau}$ as usual, we can write $Z(d)$ as

$$
\begin{aligned}
Z(d) & =16 \int_{0}^{\infty} \mathcal{D} \tau \tau k \frac{d}{d k} \log \left[\left(-\frac{2 \pi}{\log k}\right)^{-1 / 2} k^{1 / 24} \mathrm{e}^{-\pi^{2} /(3 \log k)} f(k)\right] \\
& =16 \int_{0}^{\infty} \mathcal{D} \tau\left\{-\frac{1}{4}-\frac{\pi^{2}}{12 \tau}+\frac{\tau}{24}-\tau \sum_{n=1}^{\infty} \frac{n k^{n}}{1-k^{n}}\right\}
\end{aligned}
$$

By expanding the partition function

$$
[f(k)]^{2-d}=1+(d-2) k+O\left(k^{2}\right)
$$


we see that the coefficient of the term $\tau^{1-d / 2}$ in the integral contains an expansion in powers of $k$, possibly also with some extra factors coming from the first two terms in braces in Eq. (B.3).

The leading term, $O(1 / k)$, corresponds to tachyon exchange in the loop and, as always, we just neglect it by hand. We neglect also terms $O\left(\frac{1}{k(\log k)^{a}}\right)$, where $a=1,2$, for the same reason.

The next term is of $O(1)$ in $k$, and corresponds to the exchange of massless gluons. It is given by

$$
\frac{2}{3}(d-26) \int_{0}^{\infty} d \tau \tau^{1-d / 2} \mathrm{e}^{-2 \alpha^{\prime} m^{2} \tau}=\frac{2}{3}(d-26)\left(2 \alpha^{\prime} m^{2}\right)^{d / 2-2} \Gamma\left(2-\frac{d}{2}\right)
$$

where a term with an infrared cutoff $\mathrm{m}^{2}$, reminiscent of the exponentials of Green functions that we discarded, has been added. We stress again that the field theory limit is performed by sending $\alpha^{\prime}$ to zero while keeping the ultraviolet cutoff $\epsilon$ fixed and positive.

Eq. (6.17) is obtained by expanding for small $\epsilon$ after inserting Eq. (B.5) in Eq. (6.16).

Higher order terms in Eq. (B.3), $O\left(k^{I+1}\right)$ with $I>0$, are negligible in the field theory limit. This is clearly understood in string theory remembering that the power $I$ is related to the mass of the string excitation exchanged in the loop, and to $\alpha^{\prime}$, by the formula $I=\alpha^{\prime} m_{I}^{2}$ (see also Eq. (A.10)).

\section{References}

[1] See, for example, P. Di Vecchia, "Multiloop amplitudes in string theory" in Erice, Theor. Phys. (1992), and references therein.

[2] R. R. Metsaev and A. A. Tseytlin, Nucl. Phys. B 298 (1988) p. 109.

[3] J. A. Minahan, Nucl. Phys. B 298 (1988) p. 36.

[4] T. R. Taylor and G. Veneziano, Phys. Lett. 212 B (1988) p. 147.

[5] V. S. Kaplunovsky, Nucl. Phys. B 307 (1988) p. 145, and Nucl. Phys. B 382 (1992) p. 436, hep-th/9205070.

[6] Z. Bern and D. A. Kosower, Phys. Rev. D 38 (1988) p. 1888.

[7] Z. Bern and D. A. Kosower, Nucl. Phys. B 321 (1989) p. 605.

[8] Z. Bern and D. A. Kosower, Nucl. Phys. B 379 (1992) p. 451. 
[9] Z. Bern, L. Dixon and D. A. Kosower, Phys. Rev. Lett. 70 (1993) p. 2677, hep-ph/9302280.

[10] Z. Bern, Phys. Lett. 296 B (1992) p. 85.

[11] Z. Bern and D. C. Dunbar, Nucl. Phys. B 379 (1992) p. 562.

[12] M. J. Strassler, Nucl. Phys. B 385 (1992) p. 145, hep-ph/9205205.

[13] M. G. Schmidt and C. Schubert, Phys. Lett. 331 B (1994) p. 69, hepth/9403158.

[14] K. Roland, Phys. Lett. 289 B (1992) p. 148.

[15] P. Di Vecchia, A. Lerda, L. Magnea and R. Marotta, Phys. Lett. 351 B (1995) p. 445, hep-th/9502156.

[16] E. S. Fradkin and A. A. Tseytlin, Phys. Lett. 163 B (1985) p. 123.

[17] P. Di Vecchia, F. Pezzella, M. Frau, K. Hornfeck, A. Lerda and S. Sciuto, Nucl. Phys. B 322 (1989) p. 317.

[18] P. Di Vecchia, M. Frau, A. Lerda and S. Sciuto, Nucl. Phys. B 298 (1988) p. 526.

[19] M. B. Green, J. H. Schwarz and E. Witten, "Superstring Theory", Cambridge University Press (1987).

[20] Z. Bern, D. A. Kosower and K. Roland, Nucl. Phys. B 334 (1990) p. 309.

[21] L. F. Abbott, Nucl. Phys. B 185 (1981) p. 189.

[22] L. F. Abbott, M. T. Grisaru and R. K. Schaefer, Nucl. Phys. B 229 (1983) p. 372.

[23] A.A. Tseytlin, Phys. Lett. 202 B (1988) p. 81. 